\title{
1 Context sensitivity across multiple time scales with a 2 flexible frequency bandwidth
}

3 Tamar I. Regev ${ }^{1, *}$, Geffen Markusfeld ${ }^{2}$, Leon Y. Deouell ${ }^{1,2,{ }^{* *}}$, and Israel Nelken ${ }^{1,3, * *}$

$4{ }^{1}$ Edmond and Lily Safra Center for Brain Sciences, The Hebrew University of Jerusalem,

5 Jerusalem, 9190401, Israel

$6{ }^{2}$ Dept. of Psychology, The Hebrew University of Jerusalem, Jerusalem, 9190501, Israel

$7{ }^{3}$ Dept. of Neurobiology, The Hebrew University of Jerusalem, Jerusalem, 9190401, Israel

8 * Corresponding Author, tamar.regev@mail.huji.ac.il. Current address: MIT Department of

9 Brain and Cognitive Sciences, Cambridge, MA 02139, USA, tamarr@mit.edu.

** Equal senior author contribution

12 Keywords:

13 Adaptation; EEG; N1; P2; Auditory frequency; Human auditory cortex; Computational 14 modeling

Abbreviations:

16 ERP - Event-related potentials; SOA - stimulus-onset asynchrony; $L L^{\max }$ - maximum log-

17 likelihood; ms - milliseconds; $\mathrm{s}$ - seconds 


\section{ABSTRACT}

22 Everyday auditory streams are complex, including spectro-temporal content that varies at multiple time scales. Using EEG, we investigate the sensitivity of human auditory cortex to the content of past stimulation in unattended sequences of equiprobable tones. In 3 experiments including 82 participants overall, we found that neural responses measured at different latencies after stimulus onset were sensitive to frequency intervals computed over distinct time scales. Importantly, early responses were sensitive to a longer history of stimulation than later responses. To account for these results, we tested a model consisting of neural populations with frequency-specific but broad tuning that undergo adaptation with exponential recovery. We found that the coexistence of neural populations with distinct recovery rates can explain our results. Furthermore, the adaptation bandwidth of these populations depended on spectral context - it was wider when the stimulation sequence had a wider frequency range. Our results provide electrophysiological evidence as well as a possible mechanistic explanation for dynamic and multi-scale context-dependent auditory processing in the human cortex.

\section{SIGNIFICANCE STATEMENT}

It has become clear that brain processing of sensory stimuli depends on their temporal context, but context can be construed at time scales from the recent millisecond to life-long. How do different contextual time scales affect sensory processing? We show that auditory context is integrated across at least two separate time scales, and that at both of these time scales responses dynamically adapt to a varying frequency stimulation range. Using computational modeling, we develop a rigorous methodology to estimate the time and frequency scales of context integration for separate response components. Our robust results replicated across 3 EEG experiments, and contribute to the understanding of neural mechanisms supporting complex and dynamic context integration. 
To function efficiently, sensory systems should interpret incoming stimuli in the context in which they are embedded. Contextual effects in audition are often studied using sound sequences that have some regularity, which is infrequently violated by 'deviant' sounds. These studies show that in humans, auditory event-related potentials depend on the preceding statistics of the sequence (Sussman, 2007; Garrido et al., 2013; Herrmann et al., 2015). However, processing of context is important for any stimulus sequence structure and not just for detecting regularities or change thereof. Here, we generalize contextual effects beyond deviance processing, using human EEG recordings, and modelling.

One way of efficiently representing context is by summary statistics of past stimulation. Indeed, humans can reliably report the mean pitch of several pure tones (Albrecht et al., 2012; Piazza et al., 2013), and sound textures are represented using time-averaged statistics (McDermott et al., 2013). As the environment constantly changes, estimating summary descriptors dynamically may optimize information transmission (Brenner et al., 2000; Fairhall et al., 2001). Across species and modalities, neuronal input-output functions scale with statistical properties of the stimulus distribution including mean (Dunn and Rieke, 2006; Nagel and Doupe, 2006; Dean et al., 2008; Dahmen et al., 2010), variance (Blake and Merzenich, 2002; Maravall et al., 2007; Rabinowitz et al., 2011; Herrmann et al., 2013, 2014, 2015) or higher order moments (Kvale and Schreiner, 2004; Herrmann et al., 2018, 2020). The time scales over which context influences neural activity vary widely, from tens of milliseconds to minutes (Fairhall et al., 2001; Khouri and Nelken, 2015). Presumably, this variation is necessary because the natural auditory environment contains relevant information at all of these time scales. Sensitivity of auditory neural responses to regularities established across multiple time scales was reported in single A1 neurons in animals (Ulanovsky et al., 2004), human MEG (Maheu et al., 2019) or the EEG components MMN and P2 (Costa-Faidella et al., 2011). However, all of the above studies concentrated on deviance detection.

We investigated context-dependent auditory processing not involving deviance-detection mechanisms by measuring EEG responses to tone sequences in which all stimuli were equiprobable and task-irrelevant. First, we address data from the control conditions of two experiments ( 1 and 2 ) previously published (Regev et al., 2019). We observed in these data that the N1 and P2 event-related EEG components (peaking $\sim 100$ and $\sim 180 \mathrm{~ms}$ following stimulus presentation, respectively) were sensitive to past frequencies across distinct time scales. Previous studies established that the time scale of N1 sensitivity is longer than $1 \mathrm{~s}$ (Zacharias et al., 2012; Okamoto and Kakigi, 2014; Herrmann et al., 2016). Further, the context sensitivity of the $\mathrm{N} 1$ component was well explained by adaptation models, but the P2 component was not (Herrmann et al., 2013). Importantly, the latter study used a predetermined adaptation time constant to model both N1 and P2. Here, we use a frequencyspecific adaptation model (Herrmann et al., 2013, 2014, 2015) to explain our previous results as well as a new dataset (Experiment 3), but instead of using a predetermined time constant, we develop a rigorous methodology to quantitatively estimate the effective time scales of each component from the data. Consequently, we show that auditory responses at different latencies (i.e., the latencies of the N1 and P2 components) can be explained by the same mechanism of frequency-specific adaptation, but with different adaptation time scales. Notably, the earlier component (N1) was sensitive to a longer history of stimulation than the later component (P2). Furthermore, previous studies suggested that the adaptation 
bioRxiv preprint doi: https://doi.org/10.1101/2020.06.08.141044; this version posted December 9, 2020. The copyright holder for this preprint (which was not certified by peer review) is the author/funder, who has granted bioRxiv a license to display the preprint in perpetuity. It is made available under aCC-BY-NC-ND 4.0 International license.

91 bandwidth of N1, but not of P2, dynamically adapts to the range of frequencies presented in the stimulus stream (Herrmann et al., 2013). Therefore, in Experiment 3 we manipulated the 93 overall range of frequencies in the sequences. We replicated the dynamic spectral-context94 dependent adaptation bandwidth of $\mathrm{N} 1$, and generalized it to the P2 latency. We conclude 95 that this type of adaptation, with varying bandwidths and time scales, is a general mechanism 96 shaping auditory event-related potentials.

97 


\section{Participants}

101

102

103

104

105

106

107

108

109

110

111

112

113

114

115

116

117

118

119

120

121

122

123

124

125

126

127

128

129

130

131

132

133

134

135

136

137

138

139

140

Eighty-nine healthy adults participated in all 3 experiments - 25 musicians, 29 musicians, and 35 non-musicians in Experiments 1, 2 and 3, respectively. The reason musicians participated in Experiments 1 and 2 is not relevant to the current study and is explained in (Regev et al., 2019). Participants were recruited from The Hebrew University of Jerusalem, from Bezalel Academy of Arts and Design or the Jerusalem Academy of Music and Dance, and could either receive 40 NIS ( 12 US\$) per hour or course credit for participation in the experiment. The data of 7 participants (4, 1 and 2 from Experiments 1, 2 and 3, respectively) were excluded due to technical difficulties with the recording or excessive rates of artifacts. The analysis therefore included the data of 82 participants -21 in Experiment 1 ( 7 female, mean age = 29.2 years, SD $=9$ years), 28 in Experiment 2 (15 female, mean age $=24.6$ years, SD $=3.6$ years), and 33 participants in Experiment 3 (19 females, mean age $=24.6, S D=2.9$ years old $)$. Three more participants ( 1 from Experiment 2 and 2 from Experiment 3) were later excluded from data analysis due to unclear auditory responses, resulting in 79 participants overall in the critical analysis as explained further in the methods section Data Processing. All participants self-reported normal hearing and no history of neurological disorders. The experiment was approved by the ethical committee of the faculty of social science at The Hebrew University of Jerusalem, and informed consent was obtained after the experimental procedures were explained.

\section{Stimuli and apparatus}

In all experiments, participants were seated in a dimly lit, sound-attenuated, and echoreduced chamber (C-26, Eckel) in front of a 17-inch CRT monitor (100-Hz refresh rate), at a viewing distance of about $90 \mathrm{~cm}$. The screen was concealed by a black cover, with a rectangular window in the middle $(14$ by $8.5 \mathrm{~cm})$, through which they viewed the visual display. Auditory stimuli were presented through headphones (Sennheiser HD25, having a relatively flat frequency response function in the range of frequencies used in the experiment) that was placed over the EEG cap. The experiment was run using the Psychophysics toolbox (Brainard, 1997) for MATLAB (version 2013b, MathWorks) running on a 32-bit Windows XP system. Auditory stimuli were synthesized using MATLAB. The experiment included only pure tones, each of 100 milliseconds (ms) duration with a $30 \mathrm{~ms}$ linear rise and fall ramps. Stimuli were presented at a sound pressure level that was comfortable for the participants. At the beginning of the experiment each participant adjusted the relative amplitudes of each individual tone, such that all tones had approximately the same subjective loudness.

\section{Experiment design}

Participants viewed silent black and white films while tones were presented to them through headphones. The participants could choose either "The Artist" (Michel Hazanavicius, 2011) or "The Kid" (Charlie Chaplin, 1921), both silent movies. The participants were instructed to ignore the sounds. Each tone sequence in all 3 experiments was comprised of pure tones of 5 different frequencies. The specific frequencies varied between block types (see Figure 1, upper row, for an illustration of the stimuli, and a detailed description below). To create the sequences, random permutations of the 5 tones were concatenated successively. If the first 
tone of the next random permutation was the same as the last tone of the previous permutation, the order of tones in next permutation was reversed. As a result, the order of the tones was random with three constraints: i) each tone occurred exactly in $20 \%$ of the sound presentations, ii) a repetition of the same frequency never occurred, and iii) two successive presentations of the same tone frequency were separated by no more than 8 other sounds, imposing a substantial uniformity of tone occurrences over time.

\section{Experiment 1}

Two block types from Experiment 1 of Regev et al. (2019) (which served as control conditions in the previous study) were used for the current study. Condition 1 included 5 tones: Db4, B4, G5, Eb6 and A6 (277.2, 493.9, 784, 1244.5 and $1760 \mathrm{~Hz}$, respectively). Hence, the tones spanned $2 \frac{2}{3}$ octaves (32 semitones) and the inter-neighbor intervals (frequency intervals between adjacent tones on the sequence-specific frequency axis, see Figure 1B) were 10, 8 , 8 and 6 semitones from low to high frequency. The mean frequency (computed on the logarithmic frequency axis) was that of $\mathrm{G} 5-784 \mathrm{~Hz}$. Condition 2 included: Eb4, Db5, A5, F6 and B6 (311.1, 554.4, 880, 1397 and $1975.5 \mathrm{~Hz}$, respectively). These were similar to the tones in condition 1, but all shifted 2 semitones down. Three blocks of each condition were presented and their order was counterbalanced between participants. Each block included 500 trials, 100 of each specific tone. This resulted in 300 trials for each specific tone in each condition. The tones were presented with an SOA (stimulus onset asynchrony, i.e., the time interval between the onsets of two consecutive stimuli) of either 450 or $550 \mathrm{~ms}$, randomly (average SOA was $500 \mathrm{~ms}$ ). As a result, each block lasted $250 \mathrm{~s}$, and there were at least $30 \mathrm{~s}$ of silence between the blocks (at the participant discretion). See Figure 1B for illustration of stimuli.

\section{Experiment 2}

One block type from Experiment 2 of Regev et al. (2019) (which served as a control condition in the previous study) was used for the current study. This condition included 5 tones with equal probabilities: Db4, C5, F\#5, D6 and B6 (277.9, 523.2, 740, 1174.7, and $1975.6 \mathrm{~Hz}$, respectively). Hence, the tones spanned $2 \frac{5}{6}$ octaves (34 semitones) and the inter-neighbor intervals were 11, 6, 8 and 9 semitones from low to high frequency. The mean frequency (computed on the logarithmic frequency axis) was that of $\mathrm{F} \# 5-740 \mathrm{~Hz}$. Two blocks of this condition were presented. Each block included 550 trials presented with an SOA of $400 \mathrm{~ms}$. This resulted in 220 trials for each specific tone. Each block lasted $220 \mathrm{~s}$, and there were at least $30 \mathrm{~s}$ of silence between the blocks (at the participant discretion). See Figure 1B for illustration of stimuli.

\section{Experiment 3}

In this experiment we manipulated the overall range of frequencies in the sequences (Table 1). Five block types were used. Block 1 with a wide range of frequencies (Wide; $3 \frac{1}{4}$ octaves between the lowest and highest tone, 39 semitones), Blocks $2 \mathrm{a}$ and $2 \mathrm{~b}$ with a medium range (Medium; $2 \frac{1}{3}$ octaves, 28 semitones), and blocks $3 \mathrm{a}$ and $3 \mathrm{~b}$ with a narrow range (Narrow; $1 \frac{1}{6}$ octaves, 14 semitones). The inter-neighbor intervals were 9.75, 7 and 3.5 semitones in the Wide, Medium and Narrow range conditions. For the Medium and Narrow conditions, we designed 2 different block types ( $a$ and $b$ ), transposed by 7 semitones, in order to generalize 
the results beyond the specific mean or range of frequencies (Figure 1B). Each block type was presented 3 times so the experiment consisted of 15 blocks. The order of the blocks was randomized for each participant separately, with the constraint that successive blocks had to be of different types. In every block 540 tones were presented in total, resulting in 324 trials overall for each tone frequency in each block type.

The frequencies of all tones were taken from the $\mathrm{C}$ major scale, in order to prevent a situation in which one of the tones would become harmonically deviant and therefore result in stronger ERP responses (Poulin-Charronnat et al., 2006; Koelsch, 2009).

Table 1 - Description of stimuli in Experiment 3. Tone properties are listed from low to high.

\begin{tabular}{|l|l|l|l|l|l|}
\hline Condition & $\mathbf{1}$ (wide) & 2a (medium) & 2b (medium) & 3a (narrow) & 3b (narrow) \\
\hline Notes & $\begin{array}{l}\mathrm{A} 3, \mathrm{G} 4, \mathrm{E} 5, \mathrm{D} 6, \\
\mathrm{C} 7\end{array}$ & $\begin{array}{l}\mathrm{C} 4, \mathrm{G} 4, \mathrm{D} 5, \mathrm{~A} 5, \\
\mathrm{E} 6\end{array}$ & $\begin{array}{l}\mathrm{G} 4, \mathrm{D} 5, \mathrm{~A} 5, \mathrm{E} 6, \\
\mathrm{~B} 6\end{array}$ & $\begin{array}{l}\mathrm{G} 4, \mathrm{~B} 4, \mathrm{D} 5, \mathrm{~F} 5, \\
\mathrm{~A} 5\end{array}$ & $\begin{array}{l}\mathrm{D} 5, \mathrm{~F} 5, \mathrm{~A} 5, \mathrm{C} 6, \\
\mathrm{E} 6\end{array}$ \\
\hline $\begin{array}{l}\text { Frequencies } \\
\text { (Hz) }\end{array}$ & $\begin{array}{l}220,392, \\
659.26,1174.7, \\
2093\end{array}$ & $\begin{array}{l}261.6,392, \\
587.33,880, \\
1318\end{array}$ & $\begin{array}{l}392,587.3, \\
880,1318, \\
1975.5\end{array}$ & $\begin{array}{l}392,493.88, \\
587.33,698.46, \\
880\end{array}$ & $\begin{array}{l}493.88,698.46, \\
880,1046.5, \\
1318\end{array}$ \\
\hline $\begin{array}{l}\text { Inter-neighbor } \\
\text { intervals* (low } \\
\text { to high, } \\
\text { semitones) }\end{array}$ & $10,9,10,10$ & $7,7,7,7$ & $7,7,7,7$ & $4,3,3,4$ & $3,4,3,4$ \\
\hline $\begin{array}{l}\text { Mean } \\
\text { frequency } \\
\text { (note, Hz) }\end{array}$ & $\mathrm{F5}, 698.46$ & $\mathrm{D} 5,587.33$ & $\mathrm{~A} 5,880$ & $\mathrm{D} 5,587.33$ & $\mathrm{A5}, 880$ \\
\hline $\begin{array}{l}\text { Range } \\
\text { (octaves) }\end{array}$ & $3 \frac{1}{4}$ & $2 \frac{1}{3}$ & $2 \frac{1}{3}$ & $1 \frac{1}{6}$ & $1 \frac{1}{6}$ \\
\hline $\begin{array}{l}\text { Range } \\
\text { (semitones) }\end{array}$ & 39 & 28 & 28 & 14 & 14 \\
\hline $\begin{array}{l}\text { Mean Inter- } \\
\text { neighbor } \\
\text { interval } \\
\text { (semitones) }\end{array}$ & 9.75 & 7 & 7 & 3.5 & 3.5 \\
\hline
\end{tabular}

${ }^{*}$ Mean interval between nearby tones on the sequence-specific frequency scale, Figure $1 B$

The SOA was randomly set to one of 5 possible values: $450,475,500,525$ or 550 ms. As a result, the duration of each block was about $270 \mathrm{~s}$ ( 4.5 minutes), and there were at least 45 seconds of silence between blocks (at the participant discretion). In total, the EEG was recorded for approximately an hour and a half.

\section{EEG recording and preprocessing}

EEG was recorded from 64 pre-amplified $\mathrm{Ag} / \mathrm{AgCl}$ electrodes using an Active 2 system (BioSemi, the Netherlands), mounted on an elastic cap according to the extended 10-20 system (https://www.biosemi.com/pics/cap_64_layout_medium.jpg), with the addition of two mastoid electrodes and a nose electrode. Horizontal electrooculogram (EOG) was recorded from electrodes placed at the outer canthi of the right and left eyes. Vertical EOG was recorded from electrodes placed below the center of both eyes and above the center of 
the right eye. The EEG signal was sampled at a rate of $512 \mathrm{~Hz}$ (24 bits/channel) with an online antialiasing low-pass filter set at one fifth of the sampling rate, and stored for offline analysis.

EEG preprocessing was conducted using BrainVision Analyzer 2.0 (Brain Products), and MATLAB (2016b, MathWorks). The following pre-processing pipeline was applied in all experiments: First, since we paused recording during the break between blocks, de-trending was applied using MATLAB, subtracting long-term linear trends from each block, thus zeroing the signal at beginning and end of blocks and avoiding discontinuities at the border between blocks. Then, further pre-processing was done in Analyzer, using the following pipeline: 0.1 $\mathrm{Hz}$ high-pass, zero-phase-shift 2nd order Butterworth filter; referencing to the nose electrode; correction of ocular artifacts using independent component analysis (ICA) (Jung et al., 2000) based on typical scalp topography and time course; and finally, discarding epochs that contained other artifacts. The latter part was semi-automatic - first, an algorithm marked artifacts based on predefined criteria: absolute difference between samples $>100 \mu \mathrm{V}$ within segments of $100 \mathrm{~ms}$; gradient $>50 \mu \mathrm{V} / \mathrm{ms}$; absolute amplitude $>100 \mu \mathrm{V}$; or absolute amplitude $<0.5 \mu \mathrm{V}$ for a duration of more than $100 \mathrm{~ms}$. If an artifact was detected using any of these criteria, an epoch of $200 \mathrm{~ms}$ around it was marked. Then we performed visual inspection of all data to remove or add rare artifacts that were missed or marked by mistake. Artifact rejection was performed on $30 \mathrm{~Hz}$ low-passed data, causing the artifact rejection process to be blind to high frequency noise, which did not interfere with our analysis. Then, the data was exported from Analyzer to Matlab (prior to the $30 \mathrm{~Hz}$ low-pass filter). Finally, using MATLAB, a 1-20 Hz band-pass zero-phase-shift 4th order Butterworth filter was applied to the continuous data, followed by segmentation and averaging.

\section{Data processing}

We calculated Event-Related Potentials (ERPs) locked to auditory stimulus presentation. The ERP amplitudes were measured from the midline central $\mathrm{Cz}$ electrode. This location was selected because it maximizes the $\mathrm{N} 1$ and $\mathrm{P} 2$ responses and is typically used to measure these components (e.g. as in Tremblay, Kraus, McGee, Ponton, \& Otis, 2001). The data were parsed into segments beginning $100 \mathrm{~ms}$ before the onset of tone presentation and ending $400 \mathrm{~ms}$ after tone presentation. The average amplitude of the $100 \mathrm{~ms}$ pre-stimulus time served as a baseline for amplitude measurements. ERPs were obtained for each participant, by separately averaging trials of every block type and every tone, conditioned on every possible previous tone. This resulted in 5 tones $x 4$ previous tones (because there were no repetitions) $x$ number of block types (2, 1 or 5 in Experiments 1, 2 and 3, respectively) ERPs. We then calculated the peak amplitudes of the N1 and P2 components for each ERP yielding 40, 20 or 100 measurements per participant in Experiments 1, 2 and 3 respectively.

$\mathrm{N} 1$ and $\mathrm{P} 2$ peak amplitudes were calculated in two stages. First, to minimize misidentification of peaks due to noise, we determined the peak time from the average of all presentations of each tone (i.e. not conditioned on the previous tone frequency) for each block type and participant. The resulting ERPs were based on a large number of trials and had satisfactory signal-to-noise ratio. We defined the $\mathrm{N} 1$ latency as the time of the absolute minimum (most negative) in the time window between 50 and $150 \mathrm{~ms}$ after stimulus onset and the P2 latency as the time of the absolute maximum in the time window between 130 and $250 \mathrm{~ms}$ after stimulus onset. If the peak latencies corresponded to the edge of the corresponding time 
windows, the participant was excluded from data analysis. In total, 0, 1 and 2 participants were excluded for this reason from Experiment 1, 2 and 3 respectively, leaving 79 participants overall in the analysis. We then computed the average voltage in a 12-ms window around the detected peak time for every possible combination of tone frequency with a previous tone frequency. Thus, peak latencies were determined for each tone frequency and block type regardless of previous tones, whereas the peak amplitudes were measured around these latencies, contingent on the previous tones. Overall this resulted in 4480 points for each potential type $(840,540$ and 3100 for experiments 1,2 and 3 respectively), or 8960 altogether.

\section{Statistical analysis of ERP peak potentials}

To test the effect of both long- and short-term context on N1 and P2 peak amplitudes, we used linear mixed effect models (LME). LME were run in Matlab 2016b using the fit/me function. To be able to compare between the N1 and P2 amplitudes, the data were standardized using a z-score transform on the N1 and P2 separately, after multiplying N1 data points by -1 . N1 and P2 amplitude values were modeled using 8 fixed factor predictors, 4 for each potential type. The 4 predictors were: an intercept, two continuous slope variables termed: Interval-Mean (long-term context: frequency interval between current tone and mean frequency overall in the sequence, semitones), Interval-Previous (short-term context: interval between current and previous tone frequencies, semitones) and another slope variable representing interaction between the two latter variables, encoded as their product: Interval-Mean*Interval-Previous. Random effect factors were added for all of the 8 fixed factors, grouped by participant number. In general, to determine whether a factor should be part of the model, we compared the two (nested) models trained with and without this specific factor using a likelihood ratio test (matlab compare routine). If the likelihoods of the two models were not significantly different that factor was excluded. Thus, in this model we omitted the random slope of the interaction variables Interval-Mean*Interval-Previous (both for N1 and P2) since they did not contribute to the overall explained variance (Likelihood ratio test between the model with and without these factors; $\chi^{2}(2)=3, p=0.21$ ). This resulted in 6 random effect terms in the LME. Thus, the LME model is described with the following Wilkinson formula (Wilkinson and Rogers, 1973):

$$
\begin{aligned}
\text { Voltage } \sim I(N 1) & +I(P 2)+\text { Dist }_{\text {mean }} \cdot I(N 1)+\text { Dist }_{\text {mean }} \cdot I(P 2)+\text { Dist }_{\text {prev }} \cdot I(N 1)+\text { Dist }_{\text {prev }} \\
& \cdot I(P 2)+\text { Dist }_{\text {mean }} \cdot \text { Dist }_{\text {prev }} \cdot I(N 1)+\text { Dist }_{\text {mean }} \cdot \text { Dist }_{\text {prev }} \cdot I(P 2)+(I(N 1) \mid s) \\
& \left.+(I(P 2) \mid s)+\left(\text { Dist }_{\text {mean }} \cdot I(N 1) \mid s\right)+\text { Dist }_{\text {mean }} \cdot I(P 2) \mid s\right)+\left(\text { Dist }_{\text {prev }} \cdot I(N 1) \mid s\right) \\
& +\left(\text { Dist }_{\text {prev }} \cdot I(P 2) \mid s\right)
\end{aligned}
$$

Where Voltage is either N1 or P2 standardized amplitudes of the responses to a specific tone (given all previous tone possibilities, for each experiment, condition and participant). $I(N 1)$ and $I(P 2)$ are indicator functions for $\mathrm{N} 1$ or $\mathrm{P} 2$ (each being 1 when the voltage belongs to the corresponding class and 0 otherwise). They therefore represent separate intercepts for

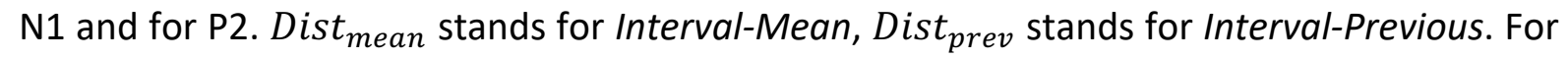
example, $\operatorname{Dist}_{\text {prev }} \cdot I(N 1)$ denotes the Dist $_{\text {prev }}$ slope variable contributing to the N1 amplitudes. $(X \mid s)$ denotes the random variable $X$ grouped by participant number. The variable $X$ is always assumed to be normally distributed with a mean of 0 , and its variance is estimated from the data. Thus, $(I(N 1) \mid s)$ denotes a subject-specific contribution to the intercept for the $\mathrm{N} 1$ measurements; $\left(\right.$ Dist $\left._{\text {prev }} \cdot I(N 1) \mid s\right)$ is a subject-specific contribution to the corresponding slope. This LME model was estimated from data points from all 3 
experiments together, resulting in 8960 data points overall (see end of Data Processing section above), collected from 79 participants.

Note that this is almost identical to modeling each potential type (N1 or P2) separately by: Voltage $\sim I+$ Dist $_{\text {mean }}+$ Dist $_{\text {prev }}+$ Dist $_{\text {mean }} \cdot$ Dist $_{\text {prev }}+(I \mid s)+\left(\right.$ Dist_prev $\left._{-} * I \mid s\right)+\left(\right.$ Dist_mean $_{\text {. }}$. $I \mid s)$.

However, there are some distinctions. For instance, in the way we estimated the model the residual error is calculated overall from all data together and therefore it is more appropriate for statistical comparisons between the estimates of N1 and P2, which was one of our main goals.

For each fixed effect coefficient, a standardized effect size, Cohen's d, was computed by dividing the estimate by its standard deviation (SD). The SD was calculated from the estimate standard error (SE) provided by fitlme, multiplying it by the square root of the number of degrees of freedom, DF (DF = 78; number of participants -1 ). The significance value of each individual coefficient (ANOVA comparing it to 0 ) was given by the fit/me model output. To statistically compare between the contributions of two (or more) coefficients we ran a posthoc coefficient test (F-test) for LME estimates, using the coefTest function in Matlab. Cohen's $d$ of these effects was calculated as the square root of $F / D F$ ( $D F=78$, as above).

To ensure the robustness of the results, we also ran a 2-level analysis, commonly used for example to analyze group results in functional MRI studies. A linear regression was run for each participant with regressors similar to the fixed effects above. We then performed a second-level analysis of the estimates using paired t-tests on participant-specific estimate values (see supplementary Figure S1 and Table S1).

To test the interaction of the overall frequency range of tones in the sequence with the effects estimated by the LME analysis described above, we ran another LME model adding interactions with the continuous slope variable range (overall frequency range in the sequence, semitones: Wide: 39, Medium: 28 and Narrow: 14). For each term in the above model we added another interaction term with range. This resulted in a large number of variables in the model and therefore we omitted the higher order interactions that did not contribute to the overall explained variance, according to a likelihood ratio test as described above. All random terms including the range variable as well as both fixed and random effects of the Interval-Mean*Interval-Previous (with and without range interaction) terms did not contribute to the overall explained variance (Likelihood-ratio test between the models with and without all of the latter terms; $\chi^{2}(14)=24.9, p>0.99$ ) and therefore were omitted from this model. However, since the fixed factors of the interaction terms Interval-Mean*IntervalPrevious were included in the previous model, we estimated as well a model including these variables and verified that the results were comparable with and without them (supplementary Table S3). This LME model was run only on data points from Experiment 3, in which the range was manipulated within subject, resulting in 6200 data points (see end of first paragraph of Data Processing section above) collected from 31 participants. Effect sizes were calculated similar to the above, using $D F=31-1=30$.

\section{Single trial EEG amplitude extraction}

We calculated single trial EEG amplitudes as the average voltage in a window of $12 \mathrm{~ms}$ centered around the latency of the N1 and P2 as determined by the subject's ERP (i.e. average 
across trials). After excluding trials with electric artifacts, we were left with 2626, 1090, 7379 observations per participant on average in experiments 1, 2 and 3, respectively $(313,366$ observations overall were used to train the model). We also fitted the model separately for each of the frequency range conditions in Experiment 3. The total number of observations used in each of these conditions was 45,714, 91,979 and 91,072, respectively. (Recall that there were 2 versions of the Medium and Narrow conditions, Figure $1 \mathrm{~A}$, hence the larger number of trials).

\section{Adaptation Model}

We used frequency-specific adaptation equations (Herrmann et al. 2013, 2014, 2015) to model single trial N1 and P2 responses. The model assumes frequency-tuned neural populations with Gaussian response profiles over a log-frequency axis. Each Population has resources, which determine the size of the responses that it can generate and are depleted in proportion to these responses. Therefore, resource depletion causes the contextual attenuation of the responses. Between sound presentations, resources recover exponentially with time. The amount of resources that are depleted is termed response adaptation (RA) following Herrmann et al. $(2013,2014,2015)$. The RA values just before the presentation of a tone were calculated recursively for each specific stimulus sequence, trial by trial, using Equation 1.

Equation 1. $R A_{i, t+1}=\left(R A_{i, t}+\left(1-R A_{i, t}\right) e^{-\frac{1}{2}\left(\frac{\log \left(f_{i}\right)-\log \left(f_{t}\right)}{\sigma}\right)^{2}}\right) \cdot e^{\frac{-\Delta t_{S_{t} \rightarrow S_{t+1}}}{\tau}}$.

Here $R A_{i, t}$ is the response adaptation of neural population i centered around frequency $f_{i}$, at time step t of the stimulus sequence, in which stimulus $S_{t}$ with frequency $f_{t}$ was presented. RA ranges between 0 (no adaptation) and 1 (maximal adaptation) and therefore 1-RA is the amount of available resources, and is therefore proportional to the response of neurons (full adaptation corresponds to minimum responsiveness and vice versa). The Gaussian term determines the amount of response evoked from population $i$ by the stimulus presented at time $t$, and therefore of additional resource depletion that is determined by the interval between the current tone presented in the sequence and $f_{i}$, the best frequency of neural population $i . \Delta t_{S_{t} \rightarrow S_{t+1}}$ is the time interval passing between the onset of the stimulus at time step $t$ and the next stimulus at time step $t+1$. During this time, resources recover and thus RA decreases exponentially. There are two parameters in Equation 1: the Gaussian width of the frequency profiles - $\sigma$, and the time constant of the exponential recovery - $\tau$. These were termed together $\vec{\theta}=(\sigma, \tau)$. Once $\vec{\theta}$ is given, Eq. 1 allows the computation of the adaptation level for each neuronal population and at each time point.

Next, the model assumes a linear relationship between the measured EEG data and RA of the population at the presented tone frequency (Equation 2).

Equation 2. Voltage $=a+b \cdot R A$,

where Voltage is the measured EEG amplitude, either N1 or P2, and $a$ and $b$ are the linear factors associating RA and the data. Since RA is defined between 0 and 1 , these factors allowed for appropriate scaling and shift to the EEG units of measurement. The inverse relation between RA and the responsiveness of neurons (1-RA) is captured by the values of $a$ and $b$. 
$R A_{i, t}$ was first computed for all the 5 tone frequencies $\mathrm{i}$ and all $\mathrm{t}$ time steps in a specific sequence. Each participant heard different stimulus sequences and therefore RA was computed for each participant separately. Then, to predict responses at each time step $t$, the relevant RA was taken as that of the i corresponding to the currently presented tone, as in Herrmann et al. $(2013,2014,2015)$. The last step assumes that EEG responses measured at time step $\mathrm{t}$ are dominated by neural population centered around the frequency of the presented stimulus $f_{i}=f_{t}$.

The procedure we used was the one used by Hermann et al. $(2013,2014,2015)$, and we used it in order to be consistent with them. A more natural procedure would consist of integrating the RA values for all neuronal populations, weighted by their tuning profiles (the exponential term in Eq. 1). We verified that the two methods are comparable by calculating the full RA predictions, weighted by the tuning profiles, for one specific participant and plotting them against the simplified model. We show that the two models are strongly linearly dependent (supplementary Figure S3).

\section{Model fit and parameter estimation}

All data analysis related to model fitting was carried out using Matlab 2016b (Mathworks, MA, USA). In total, the model had four free parameters - The two mechanistic parameters $\vec{\theta}=(\sigma, \tau)$, and the linear factors $a$ and $b$. All parameters were estimated from the data. However, $\vec{\theta}$ and the linear factors were treated differently. $\vec{\theta}$ are the parameters of interest for this study, while the linear factors are 'nuisance' parameters that have to be fitted but are not interpreted. Therefore, fitting the model was done in two steps. First, single-trial model predictions (RA) were calculated for a range of pre-defined possible $\vec{\theta}$ values; 18 values for $\sigma$ spanning 1 to 18 semitones, and 25 for $\tau$, spanning 0.2 to 5 seconds with 0.2 second steps (resulting in 450 possible $\vec{\theta}$ combinations). Second, for each $\vec{\theta}$, single trial EEG responses were regressed on the computed RA values. In practice, a linear mixed-effects model (LME) was fitted (using Matlab fit/me) using RA as a continuous fixed effect with random intercept and slopes grouped by participant (Wilkinson formula (Wilkinson and Rogers, 1973):

$$
\text { Voltage } \sim 1+R A+(1 / \text { participant })+(R A / \text { participant })) \text {. }
$$

This approach made it possible to estimate participant-specific linear factors while reducing the amount of overfitting that would be generated by estimating the linear factors of each participant separately. Log-Likelihood (LL) statistics of the LME model were extracted for each value of $\vec{\theta}$. Then, $\vec{\theta}^{\max }$, the $\vec{\theta}$ value resulting in the maximum likelihood, was selected as the best estimate for the parameters of interest.

\section{Testing significance of model fit}

To test whether the adaptation model generally described the data better than chance we repeated all the steps of parameter estimation for randomly permuted measurements. For permuted data the model was not expected to perform better than chance and therefore this allowed calculation of the distribution of the LL statistic under the null hypothesis of no effect of sequence order. We used 250 permutations. In particular, for each permutation of the data, we collected the maximum log-likelihood values (over of all possible $\vec{\theta}$ values), and plotted the null distributions of maximum LL values for N1 and P2 separately. The maximum $\mathrm{LL}$ of real data were compared to the null distributions of maximum $\mathrm{LL}$. 


\section{Confidence regions for the parameter estimates}

419 To calculate a confidence interval around $\vec{\theta}^{\text {max }}$, we asked which $\vec{\theta}$ values are significantly 420 different than $\vec{\theta}^{\max }$. Due to Wilk's theorem the quantity:

$$
D(\vec{\theta})=-2 \cdot \ln \left(\frac{\text { likelihood }(\vec{\theta})}{\text { likelihood }\left(\vec{\theta}^{\text {max }}\right)}\right)
$$

should be distributed as $\chi^{2}$ with 2 DF under the null hypothesis that $\vec{\theta}^{\text {max }}$ does not describe the data better than any other parameter value, and for a big enough sample size. We also verified this assumption empirically, simulating the null distribution of $D$ and showing that it is comparable to $\chi^{2}$ with 2 DF at 2 representative values of $\vec{\theta}$ for N1 and P2 data (supplementary Figure S4). We thus calculated the D statistic for all possible $\vec{\theta}$ values and asked whether it is significant using a $\chi^{2}$ test with 2 DF. A large D is expected for $\vec{\theta}$ values that are significantly different from $\vec{\theta}^{\text {max }}$. The statistical question of which $\vec{\theta}$ values are significantly different from $\vec{\theta}^{\text {max }}$ is equivalent to calculating a confidence region for $\vec{\theta}^{\text {max }}$. The $D$ values at $\vec{\theta}^{\text {max }}$ always equal to 0 by definition. The threshold of $D<6$, which is approximately the value corresponding to a $p=0.05$ of the $\chi^{2}$ distribution with $2 \mathrm{DF}$, was used to define the $95 \%$ confidence region around $\vec{\theta}^{\max }$.

\section{Comparing parameter estimates for $\mathrm{N} 1$ and $\mathrm{P} 2$}

We statistically contrasted the values of $\vec{\theta}^{\max }$ estimated for N1 vs. P2 using three methods. First, we compared the $\vec{\theta}^{\text {max }}$ values of $\mathrm{N} 1$ and of P2 by checking whether the $\vec{\theta}^{\text {max }}$ of, e.g. $\mathrm{N} 1$, fell outside the confidence region of $\mathrm{P} 2$ and vice versa (see previous section). Second, we performed bootstrap (random sampling with replacement) on the group of 79 participants (using all experiments together), and repeated the estimation procedure 100 times. This resulted in an estimate of the distribution of $\vec{\theta}^{\text {max }}$ values (under the assumption that the group of subjects represents the population). The distribution of differences of $\vec{\theta}^{\text {max }}$ values calculated in the same bootstrap repetition for N1 vs. P2 was compared to 0 . Third, we used data permutations to create the null distribution of the difference between $L L$ at $\vec{\theta}^{\text {max }}$ of the two potential types (termed log-likelihood differences, LLD). For each of 250 permutation repetitions, we flipped between $\mathrm{N} 1$ and $\mathrm{P} 2$ of single trials, with probability 0.5 per each trial flip. For each iteration, we repeated the estimation procedure and computed the LLD. We thus estimated the null distribution of LLD under the assumption that the parameters of N1 and P2 were identical, and calculated the p-value of the LLD of the actual data by comparing it to this null distribution.

\section{Comparing parameter estimates for different frequency ranges}

$\vec{\theta}^{\text {max }}$ values were estimated separately for each of the stimulus frequency range conditions (Wide, Medium and Small) in Experiment 3. Parameter estimation was repeated 100 times using a bootstrap procedure - simulating new groups of participants by random sampling with replacement from the pool of 31 participants. To statistically test the effect of frequency range on adaptation bandwidth of $\mathrm{N} 1$ and $\mathrm{P} 2$ we fitted an LME model to the bootstrapped $\sigma^{\max }$ values, using the Matlab fit/me function. The bootstrap repetition number was used as 
456 the grouping variable for the random effects. The stimulus frequency range variable was modeled as a continuous fixed effect with random intercept and slope. Thus, the LME model is described with the following Wilkinson formula:

$$
\begin{aligned}
\sigma^{\text {max }} \sim I(N 1)+ & I(P 2)+\operatorname{range} \cdot I(N 1)+\operatorname{range} \cdot I(P 2)+(I(N 1) \mid \text { boot } N)+(I(P 2) \mid \text { boot }) \\
& +(\text { range } \cdot I(N 1) \mid \text { boot })+(\text { range } \cdot I(P 2) \mid \text { boot })
\end{aligned}
$$

Where $\sigma^{\max }$ are the estimated adaptation bandwidths in each of 100 bootstrap runs, in each of the 3 range conditions and both for $\mathrm{N} 1$ and $\mathrm{P} 2$ data (600 values in total), and $I(N 1)$ and $I(P 2)$ are indicator functions for $\mathrm{N} 1$ or $\mathrm{P} 2$, respectively. Thus, the $I$ represent separate intercepts for $\mathrm{N} 1$ and $\mathrm{P} 2$. Range is the overall range of frequencies presented in a sequence, in semitones (so range $\cdot I(N 1)$ represents the effect of range on the $N 1$ bandwidths) and boot $N$ is the bootstrap run number serving as a grouping variable for the random effects. Effect size (Cohen's d) was calculated as above (DF=100-1=99, for 100 bootstrap repetitions). 
In 3 EEG experiments, 79 participants (21, 27 and 31 in Experiments 1, 2 and 3) were presented with sequences of 5 equiprobable pure tones (Figure $1 \mathrm{~A}$ ), which they were instructed to ignore while concentrating on a silent film. Using a passive paradigm allowed us to investigate neural effects that are elicited automatically and do not depend directly on attention or on any active task. In Experiments 1 and 2 we analyzed the control conditions from a previously published study (Regev et al., 2019) (the published study included also conditions involving rare deviants, which were the focus of that study; We do not analyze these conditions here as we focus on equiprobable sequences). The sequences from Experiment 1 and 2 we report here had a relatively wide frequency range (32 and 34 semitones between lowest and highest tones used in the sequence, respectively, Figure 1B, Methods). Experiment 3 included 3 frequency range conditions: Wide, Medium and Narrow $(39,28$, and 14 semitones respectively). We examined the amplitudes of the N1 and P2 auditory-evoked responses and their dependence on two features of the tones along the sequence: 1) The frequency interval between the current tone and the mean sequence frequency (Interval-Mean), and 2) the frequency interval between the current tone and the previous tone frequency (Interval-Previous). Interval-mean represented a long-term context variable, because in order to show sensitivity to the overall mean in the sequence, integration of several previous tones should occur (at least 5 previous tones, $\sim 2.5 \mathrm{~s}$ ). Interval-previous represented a short-term context variable, at the scale of $1 \mathrm{SOA}(\sim 0.5 \mathrm{~s})$.

\section{$488 \quad \mathbf{N} 1$ but not $\mathbf{P} 2$ is sensitive to long-term context}

Absolute $\mathrm{N} 1$ amplitudes increased as a function of the frequency interval between the current tone and overall mean frequency in the sequence. This dependence manifested itself as a typical inverted $\mathrm{U}$-shape pattern, so that the most negative $\mathrm{N} 1$ amplitudes were elicited in response to the most extreme tones and the least negative $\mathrm{N} 1$ was elicited by the middle tone (which was also approximately equal to the mean frequency of the sequence). This phenomenon was robust and replicated in all 3 experiments (Figure 1C, E). In contrast, P2 amplitudes did not show significant dependence on the mean sequence frequency (Figure 1D, E). To quantify this effect, we used a linear mixed effect model (LME) including data from all experiments together (Table 1, Figure 2). The slope of the N1 amplitudes on Interval-Mean was significantly different from $0(F(1,8952)=70, p=6.7 E-17, d=0.94$, Table 1$)$, while the slope of the P2 amplitudes on Interval-Mean was not significantly different from $0(F(1,8952)=0.15$, $\mathrm{p}=0.7, \mathrm{~d}=-0.04)$. The difference between the N1 and P2 slopes for Interval-Mean was significantly different from $0(F(1,8952)=35.5, p=2.6 E-09, d=0.67$, Table 1 , Figure 2 - contrast \#3). A two-stage procedure including linear regression on individual participants followed by Table S1). 
bioRxiv preprint doi: https://doi.org/10.1101/2020.06.08.141044; this version posted December $9,2020$. The copyright holder for this preprint (which was not certified by peer review) is the author/funder, who has granted bioRxiv a license to display the preprint in perpetuity. It is made available under aCC-BY-NC-ND 4.0 International license.

A

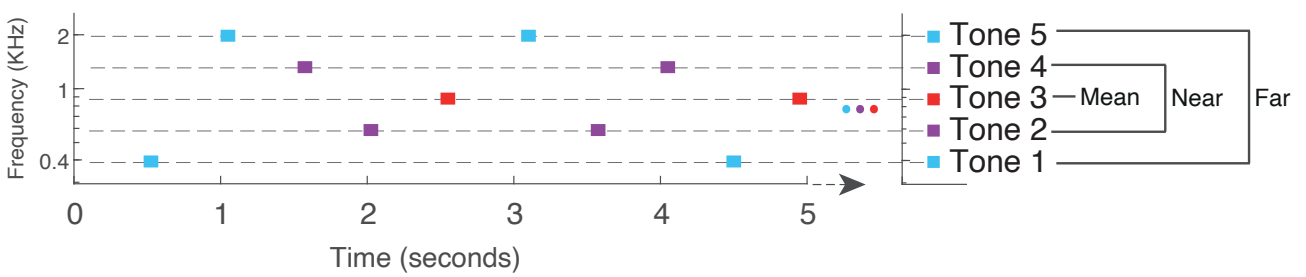

B

B Experiment 1

Experiment 2

Experiment 3

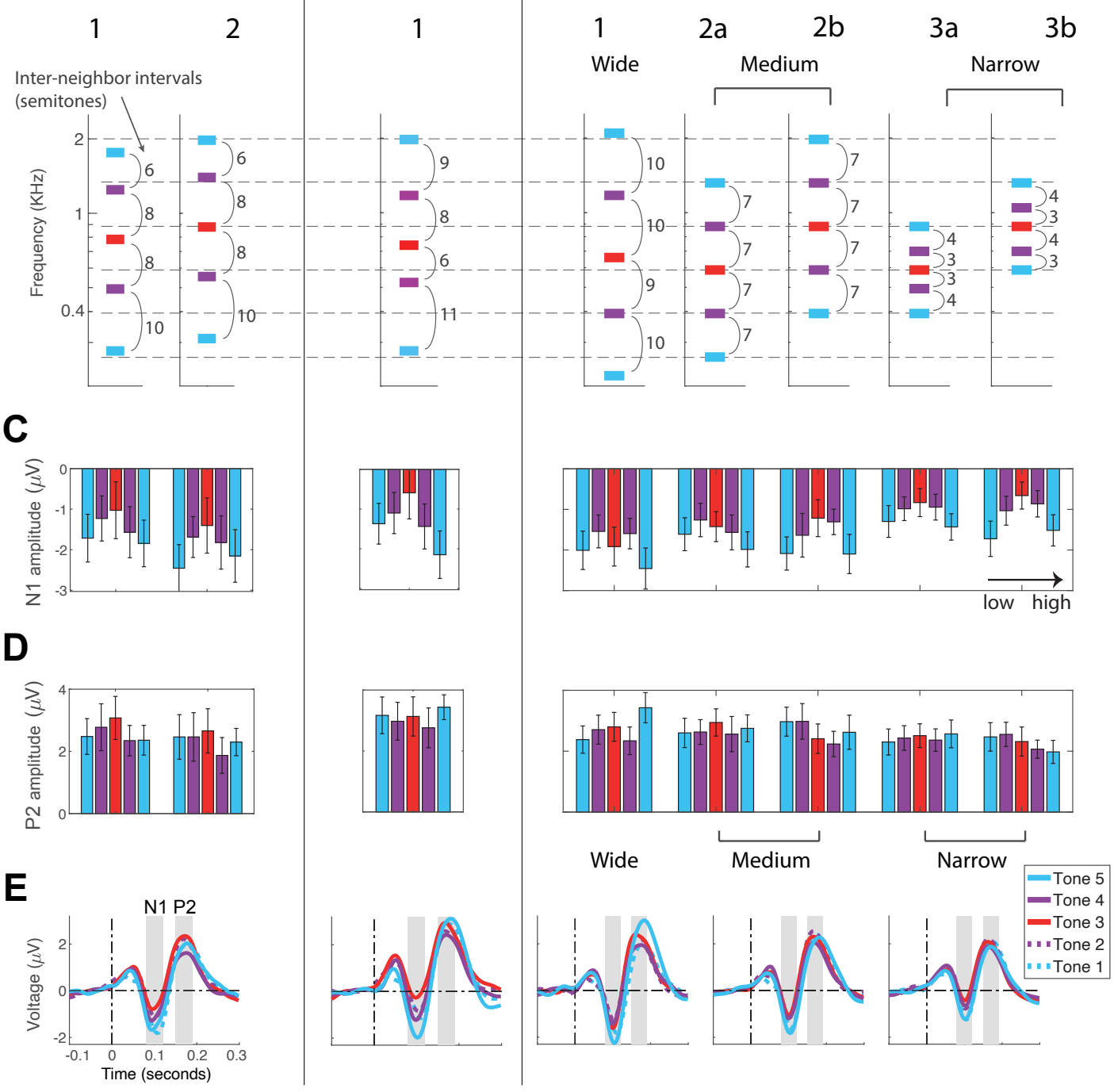

Figure 1 - N1 but not $\mathbf{P 2}$ is sensitive to long-term context. $\boldsymbol{A}-$ An example segment of a tone sequence in the experiment (from block type $2 b$ of Experiment 3 ). $\boldsymbol{B}$-Stimuli used in all experiments and conditions. Intervals between neighboring tones on the frequency axis (inter-neighbor intervals) are displayed in semitones. C-Mean and 95\% confidence intervals (across participants) of N1 peak amplitudes. For each block type the 5 bars correspond to tones 1, 2, 3, 4 and 5 (lowest to highest) from left to right and the bar colors match the color scheme in panels $A$ and B. D-Same as C for peak amplitude of P2. E-Event-related potentials (ERPs) for tones 1 to 5 (low to high frequency), calculated for each experiment. For Experiment 3, ERPs are plotted for each frequency range, pooling together block types $2 a+2 b$ and $3 a+3 b$.

Table 1 - Linear mixed effects (LME) results - effect of long-and short-term context on N1 and P2. Top - N1 and $P 2$ amplitudes (standardized using a $z$-score transform after negating N1 data points) were modeled using the 8 predictors listed in the first (left-most) column. The model consisted of fixed and random factors (grouped by participant) for each of the listed predictors (except for the interaction term Interval-Mean*Interval-Previous which did not have a random factor since the latter did not contribute to explained variance), see Methods. 
bioRxiv preprint doi: https://doi.org/10.1101/2020.06.08.141044; this version posted December 9, 2020. The copyright holder for this preprint (which was not certified by peer review) is the author/funder, who has granted bioRxiv a license to display the preprint in perpetuity. It is made available under aCC-BY-NC-ND 4.0 International license.

Columns 2 to 5: Fixed-effect estimates (Estimate), standard errors of the estimates (SE), F-statistic used for ANOVA comparing the estimates to 0 , with degrees of freedom, significance level ( $p$-value) of the latter test, standardized effect size (Cohen's d, Methods). The predictors Interval-Mean and Interval-Previous stand for the frequency interval between the current tone and sequence mean or current tone and previous tone frequency, respectively, in semitones. Bottom - Post-hoc pairwise coefficient comparison between predictors. The LME model was run on 8960 observations collected from 79 participants overall in the 3 experiments (Methods).

\begin{tabular}{|c|c|c|c|c|c|c|}
\hline \multicolumn{2}{|l|}{ Predictors } & Estimate & SE & $F(1,8952)$ & p-value & d \\
\hline \multirow[t]{2}{*}{ Intercept } & N1 & -0.51 & 0.078 & 42.2 & $8.8 \mathrm{E}-11$ & -0.73 \\
\hline & $\mathbf{P 2}$ & -0.18 & 0.074 & 5.80 & 0.016 & -0.27 \\
\hline \multirow[t]{2}{*}{ Interval-Mean } & N1 & 0.04 & 0.004 & 70.0 & 6.7E-17 & 0.94 \\
\hline & P2 & -0.002 & 0.005 & 0.15 & 0.7 & -0.04 \\
\hline \multirow[t]{2}{*}{ Interval-Previous } & N1 & 0.01 & 0.003 & 26.9 & $2.2 \mathrm{E}-7$ & 0.58 \\
\hline & $\mathbf{P 2}$ & 0.02 & 0.003 & 31.8 & $1.7 \mathrm{E}-8$ & 0.63 \\
\hline \multirow{2}{*}{$\begin{array}{l}\text { Interval-Mean * } \\
\text { Interval-Previous }\end{array}$} & N1 & -0.0008 & 0.0002 & 10.08 & 0.0015 & -0.36 \\
\hline & $\mathbf{P 2}$ & -0.0002 & 0.0002 & 0.96 & 0.32 & -0.11 \\
\hline
\end{tabular}

\begin{tabular}{|l|c|c|c|}
\hline Pairwise comparisons between predictors & $\mathbf{F ( 1 , 8 9 5 2 )}$ & p-value & d \\
\hline N1 vs. P2 Interval-Mean & 35.5 & $2.6 \mathrm{E}-9$ & 0.67 \\
\hline N1 vs. P2 Interval-Previous & 0.23 & 0.62 & -0.05 \\
\hline N1 Interval-Mean vs. -previous & 25.4 & $4.6 \mathrm{E}-7$ & 0.57 \\
\hline P2 Interval-Mean vs. -previous & 21.41 & $3.7 \mathrm{E}-6$ & -0.52 \\
\hline N1 Interval-Mean vs. -previous & & & \\
vs. & 46.3 & $1.03 \mathrm{E}-11$ & 0.77 \\
P2 Interval-Mean vs. -previous & & & \\
\hline
\end{tabular}



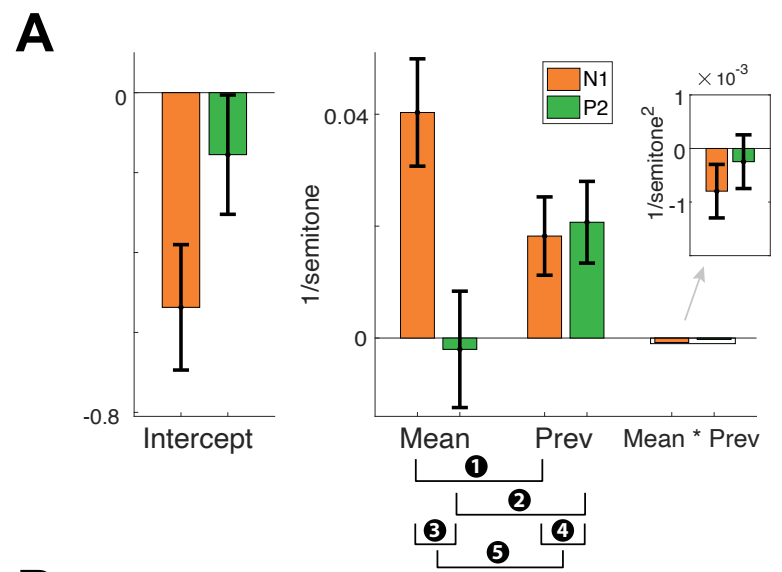

B

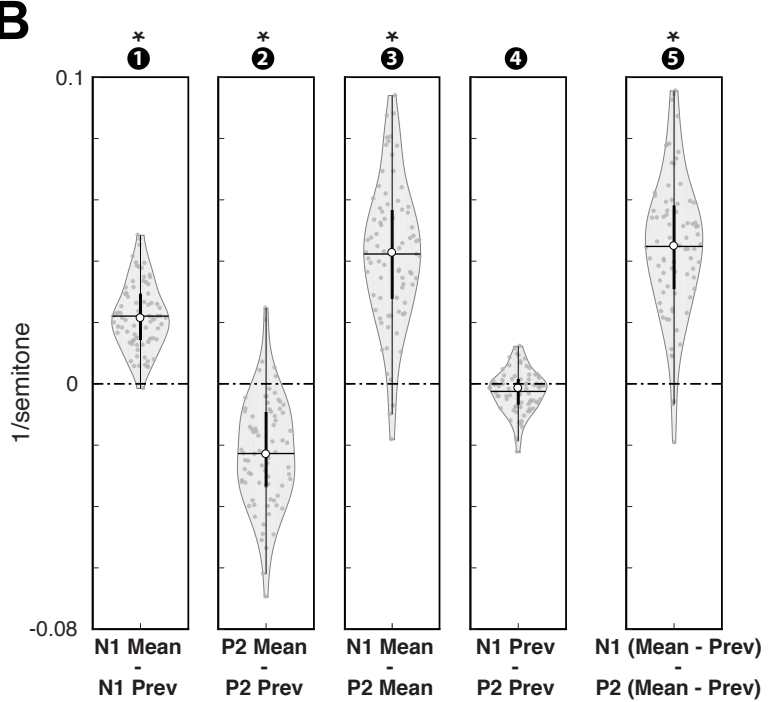

527 Figure 2 - long- and short-term context effects on N1 and P2 amplitudes. A - Bar-graphs illustrate fixed-effect estimates values from a linear-mixed effects (LME) model (Table 1 and Methods for further specification). 'Mean' and 'Prev' stand for Interval-Mean and Interval-Previous, denoting the frequency intervals between the current tone and the sequence mean or previous tone, respectively, in semitones. The predicted N1 and P2 voltages were Z-scored (after reversing the sign of the N1 data points). Error-bars represent $95 \%$ confidence intervals around the estimate (calculated by multiplying the SE of the estimate by the $95 \%$ inverse t-distribution value (DF=78)). B-Violin plots illustrate comparisons between LME estimates. Each dot represents one participant. White numbers in black circles above the violin plots indicate to which comparison they correspond (displayed under A). The significant contrasts are marked with an asterisk. Participant-specific estimates were calculated by adding the common fixed-effect estimates to participant-specific random effects. Horizontal lines represent the mean, white circles the medians, and thick and thin black vertical lines represent the $25 \%$ and 75\% percentiles, respectively.

\section{P2 is more sensitive than N1 to short-term context}

$540 \quad \mathrm{~N} 1$ and P2 absolute amplitudes increased as a function of the interval between the current and previous tone frequencies, but this effect was larger for P2 than for N1 (Figure 3). To visualize this, we pooled the possible combinations of current and previous tones according to the 'degree of neighborhood'. Neighbor 1-4 denotes the proximity of tones on the frequency axis in a specific sequence (Figure 3A, B). Figure $3(C, D)$ compares the N1 and P2 peak amplitudes for when the previous stimulus was 'Neighbor 1 ' vs. 'Neighbor 2'. We concentrated just on the 'Neighbor 1' and 'Neighbor 2' groups since they were comprised of more combinations of current and previous tones and included all current tones, whereas by design only extreme tones in every sequence could have neighbor ' 3 ' and ' 4 '. The difference 
A

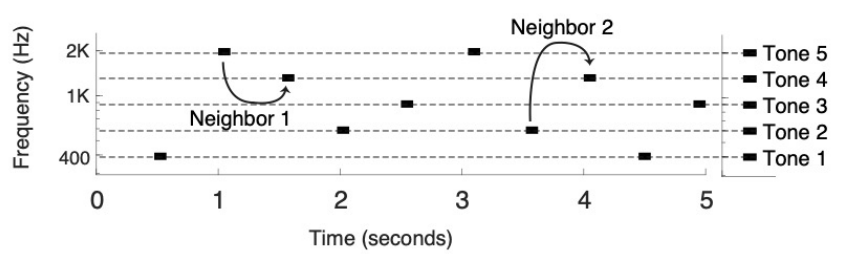

B

\begin{tabular}{|c|c|c|c|c|c|}
\hline & \multicolumn{5}{|c|}{ Current tone } \\
\hline & 1 & 2 & 3 & 4 & 5 \\
\hline 1 & & 1 & 2 & 3 & 4 \\
\hline 2 & 1 & & 1 & 2 & 3 \\
\hline 3 & 2 & 1 & & 1 & 2 \\
\hline 4 & 3 & 2 & 1 & & 1 \\
\hline 5 & 4 & 3 & 2 & 1 & \\
\hline
\end{tabular}

C
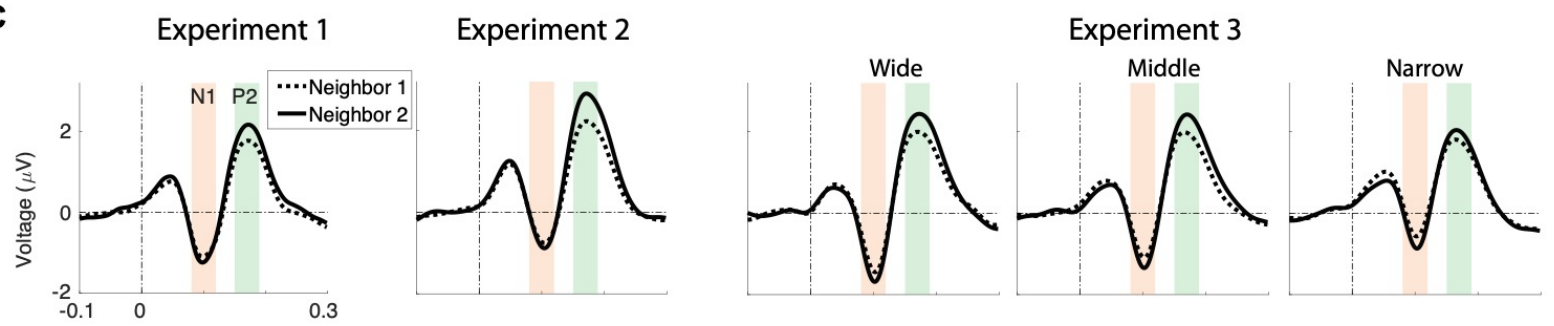

D

2
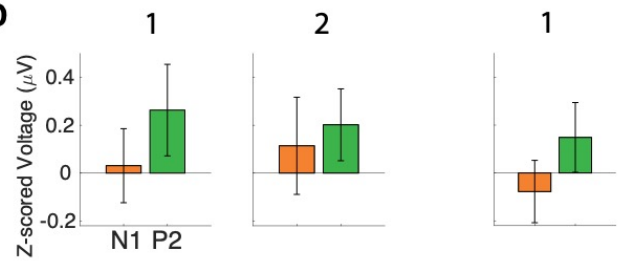

551

552

553

554

555

556

557

558

559

560

561

562

563

564

565

566

567

568

569

570

571

572

573

574

575

Figure 3 - P2 is more sensitive than $\mathbf{N} 1$ to short-term context. A - Example stimulus sequence denoting one frequency interval between two consecutive tones that are $1^{\text {st }}$ order neighbors on the sequence frequency axis ('Neighbor 1') and the $2^{\text {nd }}$ order neighbors ('Neighbor 2'). B - All possible combinations of current and previous tones in a sequence, and their grouping into the 'degree of neighborhood'. Column and row headers of the table denote ordinal tone numbers (1 to 5 from low to high frequencies, see right axis in A). Numbers inside the table denote the 'degree of neighborhood'. C - ERPs of 'current tones' when the previous tone was 'Neighbor 1' and 'Neighbor 2' for each experiment and condition separately. Shaded orange and green areas illustrate the time windows across which the N1 or P2 voltages were averaged. D - Bar-graphs denote the mean and 95\% confidence intervals (across participants) of the difference between peak amplitudes in the 'Neighbor 2' and 1' conditions. Peak amplitudes were Z-scored for N1 and P2 separately (after reversing the sign of the N1 data points).

The LME model including data from all experiments together (Table 1, Figure 2) indicated that the frequency interval between the current and previous tone (Interval-Previous, semitones) significantly affected both N1 (ANOVA comparing the LME estimates to $0 ; F(1,8952)=27$, $\mathrm{p}=2.2 \mathrm{E}-7, \mathrm{~d}=0.59$, Table 1$)$ and $\mathrm{P} 2(\mathrm{~F}(1,8952)=31.8, \mathrm{p}=1.7 \mathrm{E}-8, \mathrm{~d}=0.64)$ amplitudes. The effect size of Interval-Previous was nominally larger for P2 than for N1 but they were not significantly different $(F(1,8952)=0.24, p=0.62, d=-0.05)$. Regressions on individual participants and second-level analysis of regression estimates gave similar results (supplementary Figure S1 and Table S1). Notably, excluding the interaction term between Interval-Mean and IntervalPrevious from the LME model reduced the short-term (Interval-Previous) context effect for $\mathrm{N} 1$ but not for P2 (Supplementary Table S2), resulting in a significant difference between N1 and P2 Interval-Previous effect $(F(1,8954)=9, p=0.0025, d=-0.34)$. Thus, whereas the dependence of $\mathrm{P} 2$ on short-term context was robust, the dependence of $\mathrm{N} 1$ on short-term context interacted with its dependence on long-term context. 


\section{Sequence frequency range affects N1 but not P2 amplitudes}

$577 \quad$ N1 amplitudes were reduced when the frequency range in the sequence was smaller, while

578 P2 amplitudes were not affected much by the frequency range manipulation (Figure 1C and

579 D, Experiment 3), consistent with the fact that N1 amplitudes were more affected by long-

580 term adaptation throughout the sequence than P2. To test this statistically we ran another

581 LME model including range as a predictor (Table 2). This analysis confirmed a significant

582 contribution of range to the $\mathrm{N} 1$ intercept (ANOVA comparing LME estimates to 0;

$583 F(1,6188)=36, p=1.9 E-9, d=1.1)$ but not to the $P 2$ intercept $(F(1,6188)=1.8, p=0.18, d=0.24)$

584 and a significant difference between the effect of range on N1 and P2 intercepts $585 \quad(F(1,6188)=10.9, p=9.5 E-4, d=-0.6)$.

\section{6 \\ Sequence frequency range attenuates long- and short-term context}

587 effect

The latter LME model also indicated that both the short- and long-term context effects were attenuated for sequences with larger frequency ranges, consistent with adaptation with a limited bandwidth. There was a significant interaction between the range and the short-term context variable Interval-Previous, such that for both N1 and P2, the effect of IntervalPrevious was smaller the larger the range was $(\mathrm{N} 1: \mathrm{F}(1,6188)=4, p=4.7 \mathrm{E}-2, \mathrm{~d}=-0.36), \mathrm{P} 2$ : $F(1,6188)=6.2, p=1.2 E-2, d=-0.46)$. The interaction between range and the long-term context variable Interval-Mean was significant for $\mathrm{N} 1(\mathrm{~F}(1,6188)=7.2, \mathrm{p}=7.4 \mathrm{E}-3, \mathrm{~d}=-0.49$, smaller effect of Interval-Mean with larger range; Table 2) but not for P2 $(F(1,6188)=2.5 p=0.1, d=0.3)$. Notably, the interaction terms interval_mean*interval_previous did not contribute significantly to this model so we omitted them (Methods), however see Supplementary Table S3 for comparison to a model including these terms, which gave similar results.

Table 2 - Linear mixed effects (LME) results including interactions with frequency range. Entries are similar to observations for $N 1$ and P2 altogether (Methods).

\begin{tabular}{|c|c|c|c|c|c|c|}
\hline Predictors & & Estimate & SE & $F(1,6188)$ & p-value & d \\
\hline \multirow{2}{*}{ Intercept } & N1 & -0.76 & 0.1 & 55.3 & $1.2 \mathrm{E}-13$ & -1.4 \\
\hline & P2 & -0.36 & 0.1 & 9.7 & $1.9 \mathrm{E}-03$ & -0.57 \\
\hline \multirow{2}{*}{ Interval-Mean } & N1 & 0.054 & 0.01 & 17.3 & $3.2 \mathrm{E}-05$ & 0.76 \\
\hline & P2 & -0.028 & 0.01 & 4.2 & 4.1E-02 & -0.37 \\
\hline \multirow{2}{*}{ Interval-Previous } & N1 & 0.027 & 0.009 & 8.8 & 3.0E-03 & 0.54 \\
\hline & P2 & 0.043 & 0.009 & 21.2 & 4.1E-06 & 0.84 \\
\hline \multirow{2}{*}{ Range * Intercept } & N1 & 0.018 & 0.003 & 36.2 & 1.9E-09 & 1.1 \\
\hline & P2 & 0.0041 & 0.003 & 1.8 & $1.8 \mathrm{E}-01$ & 0.24 \\
\hline \multirow{2}{*}{ Range * Interval-Mean } & N1 & -0.0011 & 0.0004 & 7.2 & $7.4 \mathrm{E}-03$ & -0.49 \\
\hline & $\mathbf{P 2}$ & 0.0006 & 0.0004 & 2.5 & 1.1E-01 & 0.29 \\
\hline
\end{tabular}




\begin{tabular}{|l|c|c|c|c|c|c|}
\hline \multirow{2}{*}{ Range * Interval-Previous } & N1 & -0.0006 & 0.0003 & 4.0 & $4.7 \mathrm{E}-02$ & -0.36 \\
\cline { 2 - 7 } & $\mathbf{P 2}$ & -0.0007 & 0.0003 & 6.2 & $1.2 \mathrm{E}-02$ & -0.46 \\
\hline
\end{tabular}

\begin{tabular}{|l|c|c|c|}
\hline Comparisons between pairs of predictors & $\mathbf{F}(\mathbf{1 , 6 1 8 6})$ & $\mathbf{p}$-value & $\mathbf{d}$ \\
\hline N1 vs. P2 (Range * Intercept) & 10.9 & $9.5 \mathrm{E}-04$ & 0.6 \\
\hline N1 vs. P2 (Range * Interval-Mean) & 9.08 & $2.5 \mathrm{E}-03$ & -0.55 \\
\hline N1 vs. P2 (Range * Interval-Previous) & 0.13 & 0.71 & 0.06 \\
\hline
\end{tabular}

In summary, the ERP results demonstrated that N1 and P2 were affected differently by context: N1 was highly affected by long-term context (Interval-Mean) and P2 was not. Additionally, both were affected by short-term context (Interval-Previous) but P2 more robustly so. Furthermore, the spectral context (frequency range in the sequence) had a distinct effect on the $\mathrm{N} 1$ and $\mathrm{P} 2$ amplitudes: smaller sequence range reduced N1 more than P2 amplitudes, suggesting that adaptation affects the N1 more than it affects the P2. These results imply that $\mathrm{N} 1$ and $\mathrm{P} 2$ have distinct time scales of contextual influences. Additionally, the effects of long- and short-term context were generally reduced for larger frequency ranges, suggesting that some limited frequency bandwidth plays a role in these effects.

The specific values of the time scales and frequency bandwidth cannot be directly computed using the ERP analysis presented until here. Importantly, the two context predictors we used in the LMEs; Interval-Mean and Interval-Previous made it possible to consider only very short(1 previous tone) or very long-term (the sequence mean) contextual effects. In order to estimate the relevant temporal and spectral scales of these contextual effects, we employed a computational model.

\section{Adaptation model}

619 We hypothesized that both the N1 and P2 results could be generated by a single underlying neural mechanism - adaptation of frequency-selective neural populations with relatively wide bandwidths that have two different time constants.

In the auditory system, frequency-selective neurons respond not only to their best frequency but also to nearby frequencies. Therefore, presenting a tone would adapt not only neural populations tuned exactly to that tone's frequency but also populations tuned to nearby frequencies. Further, if the interval to the next tone is short enough relative to the time scale of adaptation recovery, this adaptation would not recover fully before the next stimulus occurs. Thus, given that effective frequency response profiles of neuronal populations are wider than the frequency intervals between tones in a stimulus sequence, cross-frequency adaptation (Taaseh, Yaron, \& Nelken, 2011; also termed co-adaptation, Herrmann et al., 2015; Herrmann, Schlichting, \& Obleser, 2014) would render the adapting populations sensitive to frequency intervals. Moreover, the time it takes for neurons to recover from adaptation determines the duration of this effect. If recovery rates are slow relative to the inter-stimulus interval, neurons would accumulate adaptation due to their responses to more than one previous tone in the sequence. 
635

636

637

638

639

640

641

642

643

With these premises, we used computational modelling (see Methods for equations) to test the feasibility of adaptation as the neural mechanism accounting for the ERP results presented above, and to estimate quantitatively the effective time and frequency scales underlying the context-sensitivity of the $\mathrm{N} 1$ and P2 potentials. A similar modelling approach was applied in the past for neural responses in rats (Taaseh et al., 2011). Further, this model was applied for EEG by Herrmann et al. $(2013,2014,2015)$ and we used a similar formulation to the latter studies for comparability. We fitted model predictions to single trial N1 and P2 amplitudes and estimated $\sigma$, the bandwidth of frequency response-adaptation profiles, and $\tau$, the time constant of recovery from adaptation (Figure 4), for N1 and P2 separately.

A

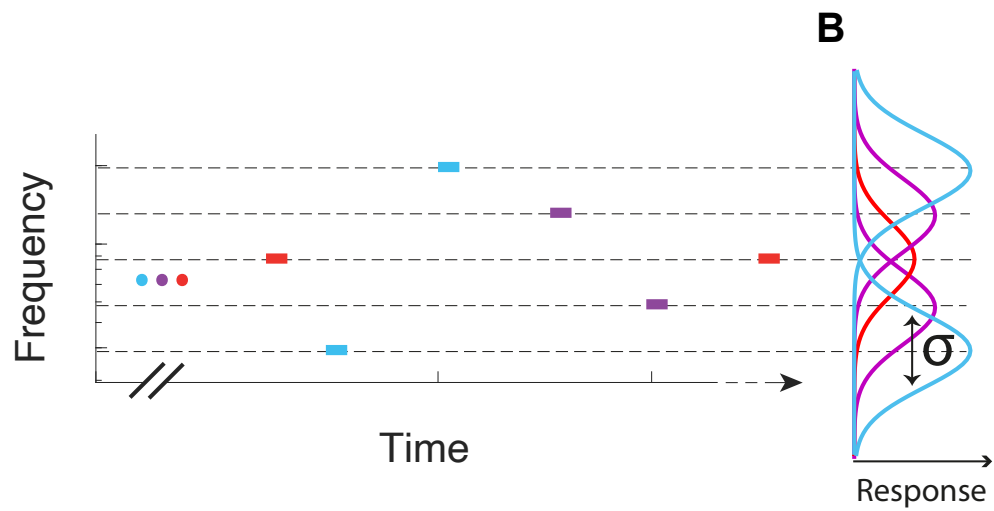

C

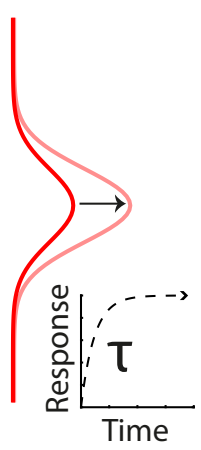

644

645

646

647

648

649

650

651

652

653

654

655

656

657

658

659

660

661

662

663

664

665

666

667

668

669

670

Figure 4 - Illustration of the adaptation model. $\boldsymbol{A}$ - An example segment of a tone sequence in the experiment serving as stimulus. Color code as in Figure 1. B - Schematic Gaussian frequency response-adaptation curves of neural populations assumed by the model. All curves have the same bandwidth $\sigma$ and each curve is centered around 1 of the 5 tone frequencies in the sequence. The color of the curves matches the color of the stimulus (A) at its best frequency. The amplitudes of the curves represent the average adaptation of the neural populations throughout the sequence (excluding 5 initial tone presentations). Populations with response-adaptation curves centered around the middle frequency (red), respond most frequently throughout the sequence and therefore have the most adapted (attenuated) response-adaptation profile. $\boldsymbol{C}$ - Exponential recovery from adaptation. The red curves represent the frequency response-adaptation curves of a population at two time points during a period with no tone presentations. Inset below is an exponential recovery function for a given time constant $\tau$. The curve increases along the arrow connecting the dark to the light red curves, as specified by the exponential function in the inset.

\section{The adaptation model accounts for both $\mathrm{N} 1$ and P2 data}

The model was fitted to single trial $\mathrm{N} 1$ and $\mathrm{P} 2$ data separately and the values of the time and frequency scale parameters, $\vec{\theta}=(\sigma, \tau)$, were estimated by selecting the $\vec{\theta}^{\text {max }}=$ $\left(\sigma^{\max }, \tau^{\max }\right)$ values maximizing the log-likelihood $\left(L L^{\max }\right)$, using a search over a predetermined grid of parameter values. To test the significance of the fit, we fitted the model to surrogate data consisting of random permutations of the measured responses across time (Methods). For both $\mathrm{N} 1$ and $\mathrm{P} 2$ the $L L^{\max }$ values obtained using the actual data were much larger than all null $L L^{\max }$ values obtained from the surrogate data. This indicated that the adaptation model fitted the data better than chance $\left(p<0.004\right.$, since real $L L^{\text {max }}$ for both N1 and P2 was larger than all $L L^{\max }$ calculated in 250 repetitions, supplementary Figure S5).

\section{N1 has a longer adaptation recovery time than P2}

The estimated time constant for recovery from adaptation, $\tau^{\max }$, was consistently longer for $\mathrm{N} 1$ relative to $\mathrm{P} 2$. This result was found when fitting the model using data from Experiments 1,2 and 3 separately, as well as when using the data from all experiments together. The values 
671

672

673

674

675

676

677

678

679

680

681

682

683

684

685

686

\section{7}

688

689

690

691

692

693

of $\tau^{\max }$ were $5,4.6,2.4$ or $3.2 \mathrm{~s}$ for $\mathrm{N} 1$ and $0.4,0.8,1$ or $1 \mathrm{~s}$ for $\mathrm{P} 2$ in Experiments $1,2,3$ or when using all data together, respectively (Figure $5 \mathrm{~A}$ ). In Experiment $1, \tau^{\max }$ of $\mathrm{N} 1$ was on the upper boundary of the allowed parameter range $(5 \mathrm{~s}$, equaling 2 repetitions of a 5 stimulus sequence). We limited the $\tau$ scale to $5 \mathrm{~s}$ since the predicted values become almost constant for larger $\tau$ values, due to the fact that the stimulus sequence was composed of successive permutations of the five frequencies. Therefore, a time constant of $5 \mathrm{~s}$ should be interpreted as 5 or longer.

The difference between $\tau^{\max }$ of $\mathrm{N} 1$ and of P2 was significant. We used three methods for comparing them (Methods): (1) The values of $\tau^{\max }$ of each potential type fell outside the $95 \%$ confidence region of $\tau^{\max }$ of the other type (Figure $5 \mathrm{~A}$ ); (2) $\tau^{\text {max }}$ of N1 was larger than $\tau^{\text {max }}$ of $\mathrm{P} 2$ in all bootstrap repetitions we performed (sampling with replacement over the 79 participants and repeating the parameter estimation procedure; Figure 5B, $p<0.01$ since 100 bootstrap repetitions were conducted); and (3) the difference between the log-likelihoods calculated at $\tau^{\max }$ and at $\tau^{\max }$ of the other potential type, were significantly larger than their null distribution, estimated by fitting the model to permuted data ( $p=0.0039$ for N1 and $\mathrm{p}=0.012$ for $\mathrm{P} 2$, supplementary Figure $\mathrm{S} 6$ ).

\section{N1 and P2 have similar frequency bandwidths of adaptation}

In contrast to the time constant, the estimated frequency bandwidth, $\sigma^{\max }$, was similar for $\mathrm{N} 1$ and for P2. $\sigma^{\max }$ was 10, 7, 8 or 8 semitones for $\mathrm{N} 1$ and 4, 9, 9, or 8 semitones for P2 in Experiments 1, 2, 3 or all together, respectively (Figure $5 \mathrm{~A}$ ). In fact, using the data of all experiments together, $\sigma^{\max }$ was exactly 8 semitones for both $\mathrm{N} 1$ and P2 (Figure 5A lowest panels). Additionally, the differences between $\sigma^{\max }$ values of $\mathrm{N} 1$ and of P2 estimated for 100 bootstrap repetitions of the 79 participants were not significantly different than 0 (Figure 5B). 
A

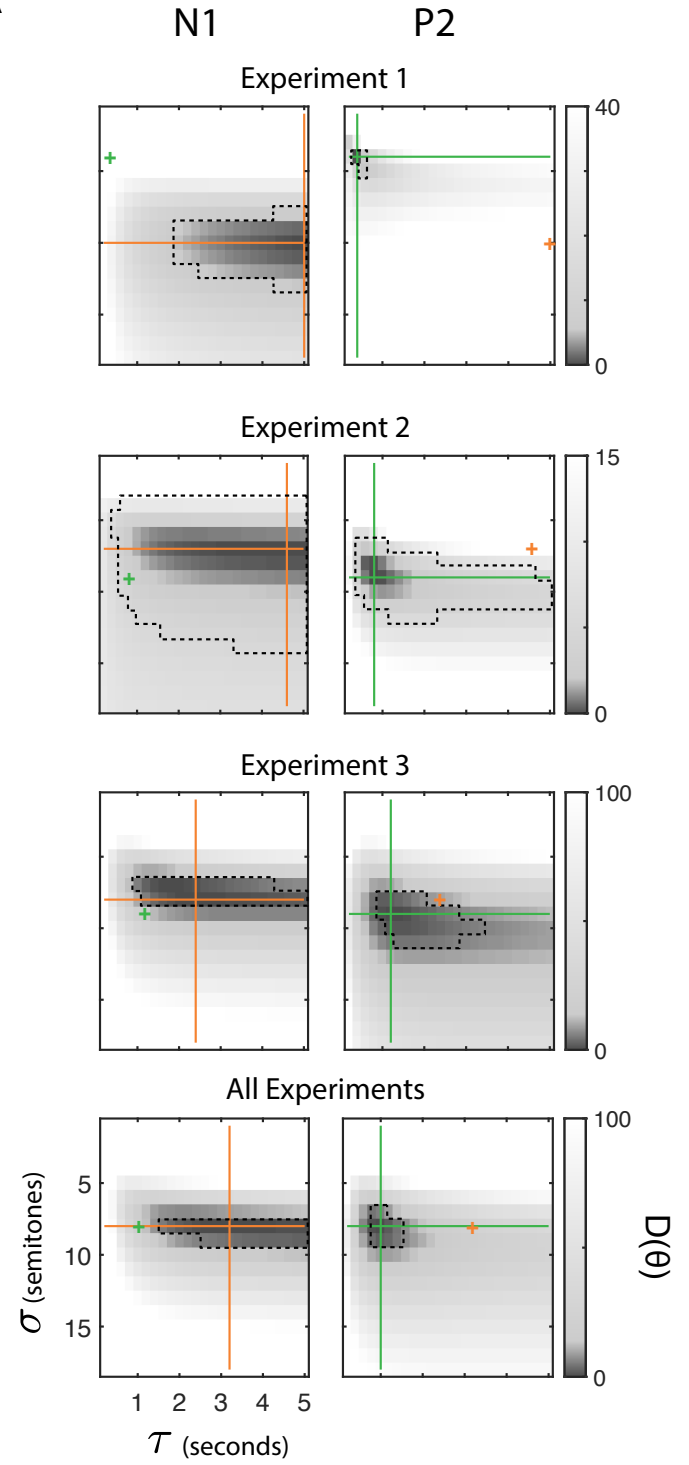

B

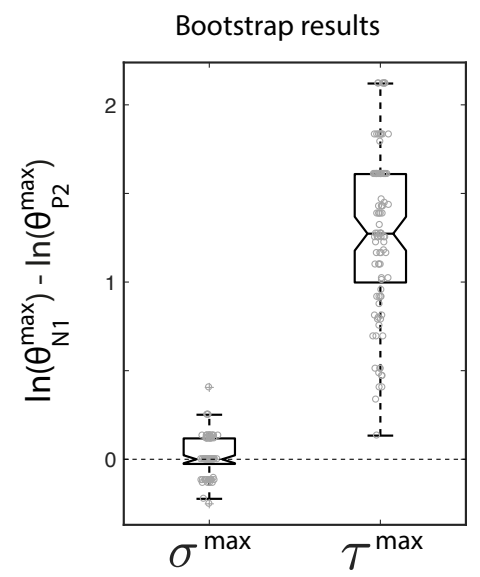

C

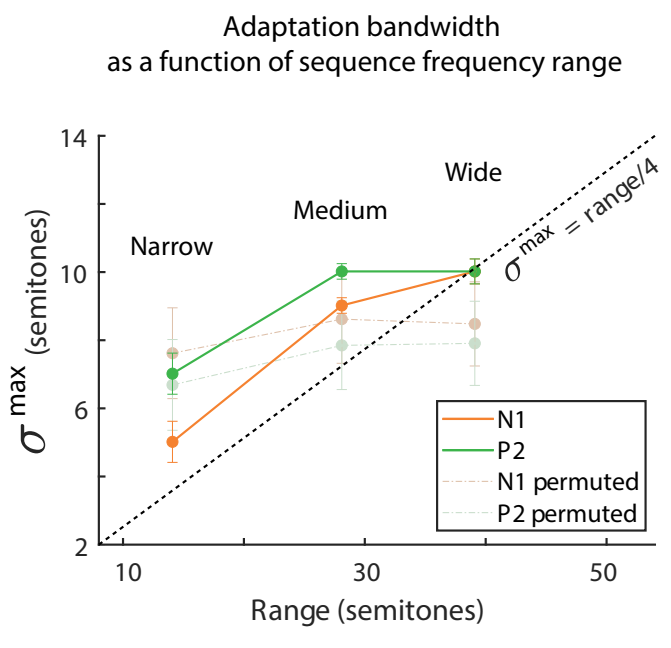

Figure 5 - Adaptation model reveals the time and frequency scales of N1 and P2 context sensitivity. A - D values (-2*log-likelihood ratio relative to $\vec{\theta}^{\text {max }}$ which maximizes the likelihood, Methods), for each possible value of $\vec{\theta}=(\tau, \sigma)$, for each experiment, for N1 (left) and P2 (right). Cross-hairs (N1: orange, P2: green) are located at the maximum-likelihood estimated parameter values (i.e. at $\vec{\theta}^{\text {max }}$ ). Small cross-signs are located at $\vec{\theta}^{\text {max }}$ of the other potential type (exactly the crossing point of the neighboring plot, same color code), for visual comparison between $\vec{\theta}^{\text {max }}$ of the two potential types. Dashed lines surround 95\% confidence regions for $\vec{\theta}^{\text {max }}$ calculated according to the null distribution of $D$ ( $\chi^{2}$ with $2 d f$, Methods). B - Bootstrap results comparing estimated parameter values for $N 1$ and $P 2$. Each gray dot is the difference between $\vec{\theta}^{\text {max }}$ estimated for $N 1$ and for $P 2$ in one of 100 bootstrap repetitions (sampling with replacement from the 79 participants and repeating the full parameter estimation procedure, Methods). $\boldsymbol{C}$ - Frequency bandwidth depends on spectral context. $\sigma^{\max }$ as a function of stimulus frequency range for $N 1$ (orange), $P 2$ (green) and for N1 and P2 after permuting the order of trials within each range condition 250 times (dashed, see legend). Data from Experiment 3 only. Error bars of the $N 1$ and $P 2$ plots are 95\% confidence intervals estimated from 100 bootstrap repetitions sampling with replacement the 31 participants and repeating parameter estimation procedure. Error bars for the permuted data plots are $95 \%$ confidence intervals calculated from the 250 permutations. 


\section{Adaptation bandwidth rescales to the sequence frequency range}

713 Next, we asked whether the parameters of the model $\left(\sigma^{\max }\right.$ and $\tau^{\max }$ ) are constant when

714 the spectral context changes. We fitted the model and estimated parameter values separately

715 for each of the frequency range conditions in Experiment 3. First, we tested whether allowing

716 the time constant, $\tau^{\max }$, to vary for different ranges improves significantly the model fit.

717 Estimating a separate $\tau$ did not increase significantly the explained variance neither for $\mathrm{N} 1$

718 (Likelihood ratio test comparing models with constant or varying $\tau: \chi^{2}=0, \mathrm{df}=2, \mathrm{p}=1$ ) nor for

$719 \mathrm{P} 2\left(\chi^{2}=4.7, \mathrm{df}=2, \mathrm{p}=0.09\right)$. We therefore estimated a single time constant for all the possible

720 ranges. Next, we tested whether the frequency adaptation bandwidth, $\sigma^{\max }$ was constant or

721 differed by spectral context. Estimating a separate $\sigma$ for each range condition significantly

722 contributed to overall explained variance for N1 (Likelihood ratio test comparing models with

723 constant or varying $\left.\sigma: \chi^{2}(2)=16.4, \mathrm{p}=0.0003\right)$, but not for $\mathrm{P} 2 \quad\left(\chi^{2}(2)=1.9, \mathrm{p}=0.38\right)$.

724 Nevertheless, we decided to fit a model with separate $\sigma^{\max }$ to both the N1 and P2 data for

725 comparison purposes. We found that, interestingly, the values of $\sigma^{\max }$ were consistently

726 close to range/4 (Figure 5C, diagonal dashed line) which is the mean interval between

727 neighboring tones on the sequence-specific frequency axis (since there are 5 possible tone

728 frequencies and 4 frequency intervals between them).

729 To additionally test whether the modulation of $\sigma$ by range was significant, we performed 101

730 bootstrap repetitions (sampling with replacement from the 31 participants) and analyzed the resulting $\sigma^{\text {max }}$ using an LME model (fixed factors: Intercept and range, random factors: Intercept and range grouped by bootstrap number, Methods). Table 3 shows that the dependence of the bandwidth on the range was highly significant with large effect sizes, for both $\mathrm{N} 1$ and $\mathrm{P} 2$. Thus, although introducing separate bandwidths for the different ranges did not improve the overall fit of the model to the P2 data, nevertheless the bandwidths estimated separately for each range were highly stable. In particular, this test provides evidence that even for the P2 data, the adaptation bandwidth at the smallest range was significantly smaller than at medium and large range (Figure $5 \mathrm{C}$, Table 3 ).

The dependence of $\sigma^{\max }$ on the stimulus frequency range could potentially be caused merely by an overall reduction of peak amplitudes in sequences with smaller ranges, without any relation to the specific order of the tones in the sequence. To test if this is the case, we permuted the order of data trials within each range condition separately, and repeated the parameter estimation procedure 100 times. The values of $\sigma^{\max }$ obtained using the permuted data showed a slight modulation by frequency range (Figure 5C, pale dashed lines) but it was not significant (slope of $\sigma^{\max }$ as a function of frequency range estimated for permuted data, $\mathrm{N} 1: 0.04,95 \% \mathrm{Cl}=(-0.030 .11)$, not different from $0: \mathrm{t}(298)=0.99, \mathrm{p}=0.32, \mathrm{P} 2: 0.05,95 \% \mathrm{Cl}=(-$ $0.02,0.12), t(298)=1.37, p=0.17)$. Furthermore, the slopes of $\sigma^{\max }$ as a function of frequency range were significantly larger when using the real and bootstrapped data compared to using the permuted data (Figure $5 \mathrm{C}$ ) both for $\mathrm{N} 1$ (slopes as a function of range: $0.21,95 \% \mathrm{Cl}=(0.19$ $0.24)$, note that this confidence region did not overlap with the confidence region of the slope for permuted data presented above) and for $\mathrm{P} 2(0.13,95 \% \mathrm{Cl}=(0.10 .15))$. Thus, the overall 
bioRxiv preprint doi: https://doi.org/10.1101/2020.06.08.141044; this version posted December $9,2020$. The copyright holder for this preprint (which was not certified by peer review) is the author/funder, who has granted bioRxiv a license to display the preprint in perpetuity. It is made available under aCC-BY-NC-ND 4.0 International license.

752 reduction of amplitudes in sequences with smaller ranges was not sufficient to explain the spectral context effect.

Table 3-LME results - effect of frequency range on adaptation bandwidth. The data consisted of 101 bootstrap estimates of adaptation bandwidth (sampling with replacement from the 31 participants of Experiment 3 and repeating parameter estimation procedure) for each range condition and potential type (606 data points). The model consisted of fixed and random factors (grouped by bootstrap number) for each of the listed predictors (Methods). Column headers are similar to Tables 1 and 2.

\begin{tabular}{|l|l|l|l|l|l|l|}
\hline \multicolumn{2}{|l|}{ Predictors } & Estimate & SE & $\mathbf{F ( 1 , 6 0 2 )}$ & p-value & d \\
\hline \multirow{2}{*}{ Intercept } & N1 & 2.7 & 0.37 & 52 & $1.7 \mathrm{E}-12$ & 0.72 \\
\cline { 2 - 8 } & P2 & 5.7 & 0.39 & 218 & $2.7 \mathrm{E}-42$ & 1.5 \\
\hline \multirow{2}{*}{ Range } & N1 & 0.22 & 0.013 & 283 & $2 \mathrm{E}-52$ & 1.7 \\
\cline { 2 - 8 } & P2 & 0.13 & 0.013 & 95 & $5.8 \mathrm{E}-21$ & 0.97 \\
\hline
\end{tabular}




\section{DISCUSSION}

763 Our results suggest neural sensitivity to past stimulation at two distinct time scales. Specifically, we show that a longer integration time scale ( $3 \mathrm{~s})$ is evident in the ERPs early after stimulus onset, at a latency of about $100 \mathrm{~ms}$ (N1), and is reflected in apparent sensitivity to the mean tone frequency. A shorter integration time scale $(\sim 1 \mathrm{~s})$ is evident at a longer latency after stimulus presentation, about $200 \mathrm{~ms}$ (P2) and is reflected in sensitivity to the immediately preceding stimulus. Further, we show quantitatively that both of these response components can be accounted for by frequency-specific adaptation of neural populations having distinct time constants of recovery. Finally, by varying the frequency range of the stimulus sequence we show that spectral context affects the frequency adaptation bandwidth of these neural populations, independent of integration times.

\section{Context integration across several time scales}

The N1 and P2 auditory evoked potentials were among the first recorded human EEG responses (Davis, 1939), but their neural generators and the computations underlying these responses are still not fully understood (Picton, 2011; Lanting et al., 2013), although their generators are thought to reside mainly in auditory cortex. N1 is well-known for being strongly attenuated by stimulus repetition, an effect termed adaptation, habituation or refractoriness (Crowley and Colrain, 2004; Picton, 2011). Previous studies have established that the time scale of $\mathrm{N} 1$ adaptation is longer than about $1 \mathrm{~s} \mathrm{(Zacharias} \mathrm{et} \mathrm{al.,} \mathrm{2012;} \mathrm{Okamoto}$ and Kakigi, 2014; Herrmann et al., 2016). N1 adaptation in a sequence of pure tones was well accounted for by the frequency-specific adaptation model we used here (Herrmann et al., 2013, 2014).

In contradistinction, the effect of stimulus repetition on P2 is more controversial. P2 was sometimes suggested to be less affected by adaptation than N1 (Crowley and Colrain, 2004; Herrmann et al., 2013, 2016) and sometimes more (Lanting et al., 2013). Importantly, previous studies failed to account for $\mathrm{P} 2$ responses using adaptation models (Herrmann et al., 2013, 2016), including exactly the same frequency-specific adaptation model we used here (Herrmann et al., 2013). However, Herrmann et al. (2013) used a single, predetermined, time constant for both N1 and P2 when fitting the model to the data $(1.8 \mathrm{~s}$, based on Sams et al., 1993). Here, by developing a methodology to directly estimate the time constant from the data, we show that both $\mathrm{N} 1$ and $\mathrm{P} 2$ responses can be well explained by frequency-specific adaptation mechanisms, but that P2 has a shorter time scale of adaptation than N1. Our results contribute to characterizing the functional distinction between the N1 and P2 responses and thus support the claim that they are generated by distinct neural populations (Knight et al., 1980; Hari et al., 1982; Lanting et al., 2013).

The time constants we report may depend on specifics of the paradigm we used. For example, the sequences we used did not contain long-term information over time scales longer than about 5 seconds. For this reason, we only tested recovery time constants up to 5 seconds. In Experiment 1 the time constant estimated for $\mathrm{N} 1$ reached the maximal value of 5 seconds (Figure 5A). This may indicate that the time scale of $\mathrm{N} 1$ sensitivity may be even longer than what we report here. Indeed, other human electrophysiology studies used sequences that contained longer-term structure and reported longer time scales of integration, 10 s, for N1 (Herrmann et al., 2016). Furthermore, Costa-Faidella et al. (2011) found that in pure tone 
sequences, P2 and the MMN were sensitive both to regularities established across short $(<1$ second) and long ( 10 seconds) time scales. It could be that sequences embedding longerterm structure can reveal longer time scale sensitivity of P2. However, the long-term structure in Costa-Faidella et al. was the violation of a globally established regularity. Thus, the reported long-term sensitivity of P2 may reflect deviance-detection and prediction mechanisms, whereas the effects we report here may be due to adaptation mechanisms that are not specifically related to regularity extraction.

\section{Early processing of long and later processing of short time scales}

Interestingly, we found that the earlier response component, N1, had a longer time constant compared to $\mathrm{P} 2$, which peaks on the scalp later. Similarly, early auditory processing of longscale sequence properties and later processing of short-scale properties was recently reported in a MEG study (Maheu et al., 2019). These results are surprising because longer time scales of integration are frequently associated with a higher-level of processing, which is expected to take place later in the processing hierarchy. For example, studies employing 'global-local' paradigms found that early sensory cortex was sensitive to short-term, local regularities whereas later and more widespread activity was sensitive to long-term regularities (Bekinschtein et al., 2009; Dürschmid et al., 2016). However, the distinction between short- and long-term regularities in the latter studies, established in the context of deviance detection paradigms, are likely to reflect neural mechanisms that are distinct from the ones underlying our results, obtained in a passive paradigm with no regularities or deviance.

One can interpret our results in terms of resolution, associating a longer scale with coarser temporal resolution and a shorter scale with a higher resolution that allows to process finer details. This view corresponds with theories such as the frame-and-fill model developed for vision (Bar, 2006; Snyder et al., 2012), or reverse hierarchy theory initially also developed for vision (Ahissar and Hochstein, 2004) but later extended to audition (Nahum et al., 2008). By these theories, we quickly obtain a general gist of the sensory scene at a coarse resolution, requiring long-scale averaging. Later on, the perceptual system may gradually resolve the details, expressed in the shorter temporal scales of processing at later stages, consistent with our results.

\section{Sensitivity to the mean}

Temporal averaging plays an important role in perception (Hollingworth, 1910; Haberman et al., 2009; Albrecht and Scholl, 2010; McDermott and Simoncelli, 2011; McDermott et al., 2013). Our results manifest modulation of neural responses due to the mean of previous stimuli: N1 responses were most adapted for stimuli closest to the mean, consistent with previous reports (Ulanovsky et al., 2004; Herrmann et al., 2013, 2014). Although we did not measure perception in this study, the neural machinery described here may be related to the neural computation of mean and its influence on perception. For instance, judgements in a frequency discrimination task were biased towards the mean of past stimuli (Raviv et al., 2012; Lieder et al., 2019). This perceptual bias to the mean was correlated to ERP adaptation (Jaffe-Dax et al., 2017), potentially linking it with the neural effects we study here. We suggest that the mean is represented by the adaptation level of a frequency-specific, yet wideband, neuronal population that adapts momentarily to stimuli as they come, then recover with a long time constant. The frequency corresponding to the most adapted population is the mean 
frequency of the sequence. This results in a time-dependent estimate of the mean frequency, which effectively averages past stimuli in a sliding window whose duration depends on the time constant of recovery.

\section{Frequency bandwidth rescales to spectral range}

We found that adaptation bandwidths rescale to the range of frequency distributions in the sequence. Our results are consistent with previous reports for N1 (Herrmann et al., 2013, 2014, 2015) and generalize them for P2 as well. This suggests that sensitivity of adaptation bandwidths to the spectral context is a general feature of auditory cortex, independent of the integration time scales. Somewhat similar effects, although in the context of deviance detection, have been shown by Garrido et al. (2013).

What could be the mechanism of this sensitivity to the spectral context? Dynamic adjustment of neural input-output functions due to changes in statistical properties of preceding stimulation has been directly shown using neural measurements in non-human animals for various perceptual dimensions such as light intensity (Dunn and Rieke, 2006), visual motion (Brenner et al., 2000) and whisker motion in the rat barrel cortex (Maravall et al., 2007). In the auditory modality, rescaling of neuronal tuning to varying statistical properties of sounds were reported in the bird midbrain (Nagel and Doupe, 2006), mammalian inferior colliculus (Kvale and Schreiner, 2004; Dean et al., 2008; Dahmen et al., 2010) and primary auditory cortex (Blake and Merzenich, 2002; Gourévitch et al., 2009; Rabinowitz et al., 2011). Thus, the change of neural response patterns due to stimulation statistics could reflect activity from the same neural population that undergoes rapid changes to network connectivity (Arnsten et al., 2010; Rabinowitz et al., 2011). Alternatively, the changes of neuronal response characteristics we measured following different stimulation ranges may also reflect recruitment of distinct neuronal populations, each with its own fixed, distinct spectral and temporal response properties (Lee et al., 2016; Osman et al., 2018).

In conclusion, we find distinct responses in the auditory cortex which are sensitive to the context over shorter and longer time scales, and which quickly and automatically adjust to the spectral context. We show that the temporal effects are likely due to different rates of release from adaptation. The mechanism of the spectral effect remains to be determined.

(1) 


\section{Acknowledgements}

889

890

891

892

893

894

895

896

897

898

899

900

901

902

903

904

905

906

907

908

909

910

We thank research assistants who aided with EEG data acquisition and preprocessing: Michal Rabinovits, Eden Krispin, Anael Benistri. TIR was supported by the Hoffman Leadership and Responsibility Program at the Hebrew University. LYD was supported by the Israel Science Foundation and Jack $\mathrm{H}$. Skirball research fund. IN was supported by a grant from the Israel Academy of Sciences (390/13) and by AdERC grant 340063 (project RATLAND), and is the Milton and Brindell Gottlieb Chair in Brain Science. The funders had no role in study design, data collection and analysis, decision to publish, or preparation of the manuscript.

\section{Author contributions}

TIR formulated the study, conducted experiments, developed and conducted analysis methods, and wrote the first draft of the paper. GM conducted experiments. IN and LYD jointly supervised the study, and participated in the design of the experiments, formulation and application of analysis methods and writing the paper.

\section{Declaration of interests}

The authors declare no competing interests. LYD declares he is a co-founder and advisor of InnerEye Ltd.

\section{Data and Code Availability}

Data and code are available via Open Science Framework (OSF): $\underline{\text { http://osf.io/mswhv }}$ 
A
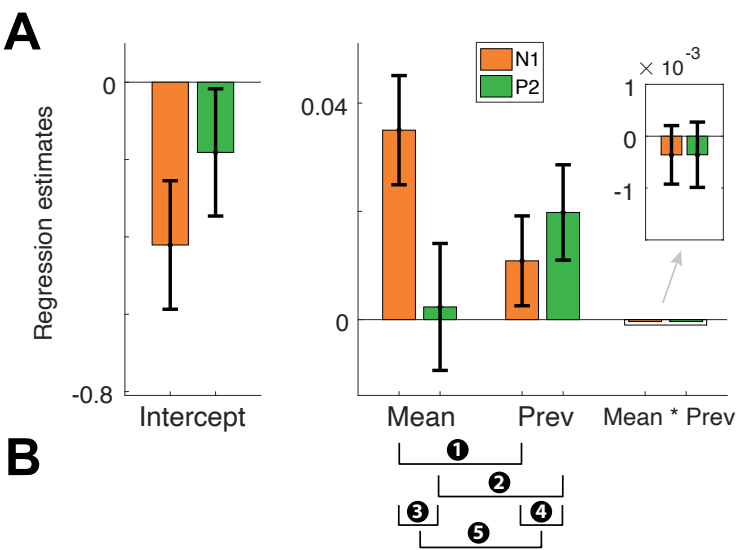

C

B

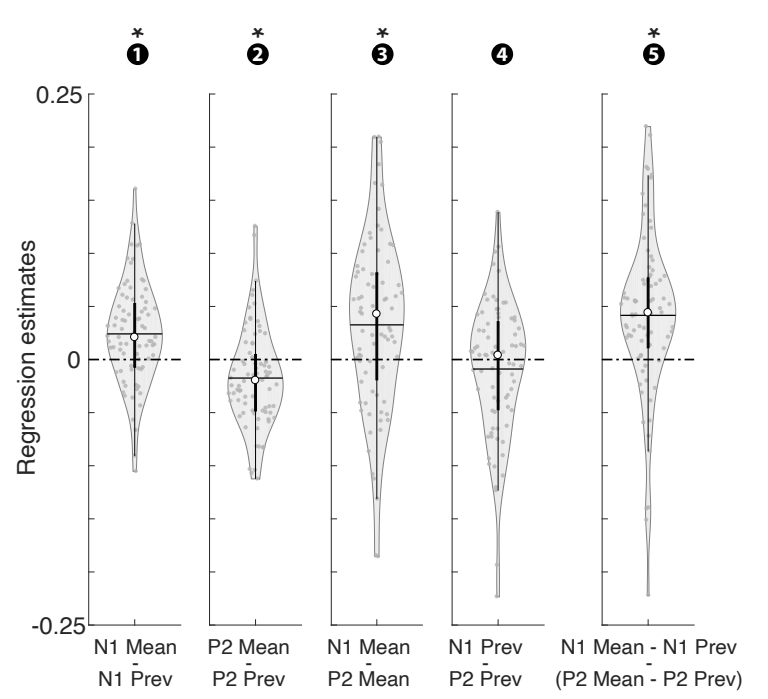

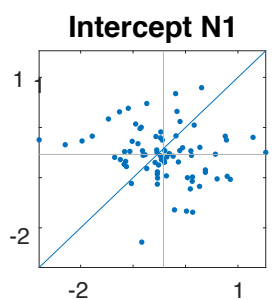
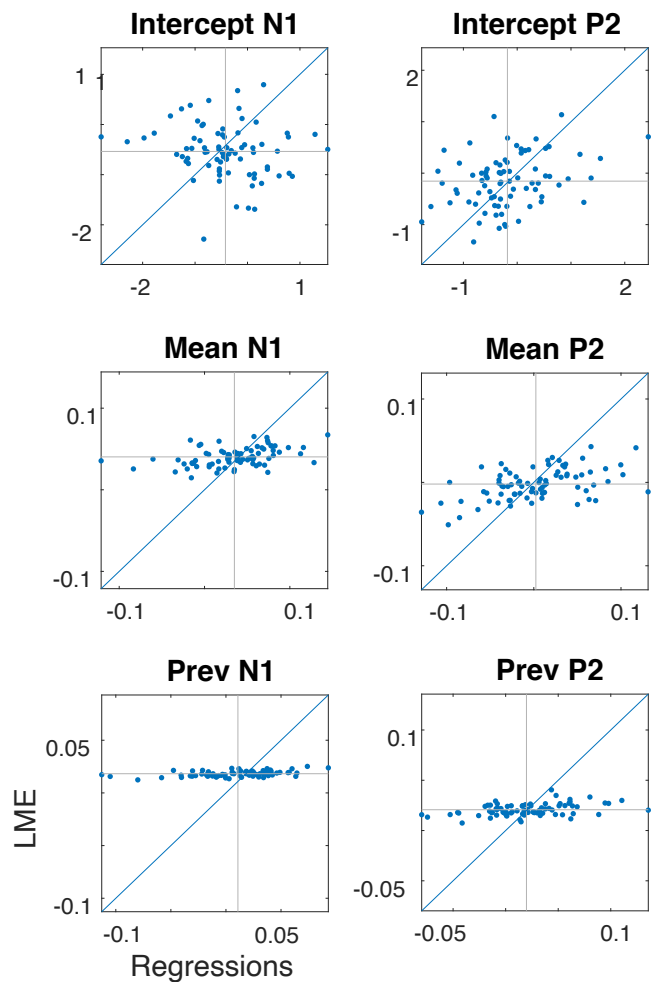

Supplementary Figure S1 - Second-level analysis of the estimates from single-participant regressions.

Compare with the results of the linear mixed effects model (LME) in Figure 2. $\boldsymbol{A}$ and $\boldsymbol{B}$ are the same type of plots as in Figure 2, but using the estimates from single-participant regressions instead of the LME estimates. For the regressions, the same predictors were used as in the fixed factors of the LME. The error bars in A represent the $95 \%$ confidence intervals calculated across the group of participant-specific estimates. Each dot in B represents the difference between the relevant estimates per each participant. Horizontal lines represent the mean, white circles the medians and thick and thin black vertical lines represent the $25 \%$ and $75 \%$ percentiles, respectively. $C$ - Scatter plots of LME vs. regression estimates per participant. Participant-specific estimates in the LME were calculated by adding the common fixed-effect estimate to the participant-specific random estimate. The fact that 
bioRxiv preprint doi: https://doi.org/10.1101/2020.06.08.141044; this version posted December 9,2020 . The copyright holder for this preprint (which was not certified by peer review) is the author/funder, who has granted bioRxiv a license to display the preprint in perpetuity. It is made available under aCC-BY-NC-ND 4.0 International license.

Supplementary Table S1 - Second-level analysis of participant-specific regression estimates, for the long-and short-term context predictors - 'Interval-Mean' and 'Interval-Previous'. N1 and P2 amplitudes (voltage in $\mu V$ was standardized using a z-score transform) modeled using the 8 predictors as in Table 1 . Here a simple linear regression was run separately for each participant (only fixed, no random factors). For each predictor the estimates for all 79 participants were collected. First row, columns 2 to 5: Estimate - mean estimate across participants. SE-standard error across the group. Cohen's $d-$ mean estimate/SD. P-value-calculated from a ttest comparing the estimate to 0 . Five bottom lines refer to a paired t-test comparing between groups of estimates. $X$ vs. $Y$ stands for a subtraction between $X$ and $Y$. In parenthesis - values from the corresponding $L M E$ model (Table 1) for comparison, when relevant.

\begin{tabular}{|c|c|c|c|c|c|c|}
\hline Predictors & & Estimate & SE & $t(78)$ & p-value & d \\
\hline \multirow[t]{2}{*}{ Intercept } & N1 & $\begin{array}{c}-0.42 \\
(-0.51)\end{array}$ & $\begin{array}{c}0.084 \\
(0.078) \\
\end{array}$ & -5 & $\begin{array}{c}2.9 \mathrm{E}-6 \\
(8.8 \mathrm{E}-11)\end{array}$ & $\begin{array}{l}-0.57 \\
(-0.74) \\
\end{array}$ \\
\hline & P2 & $\begin{array}{c}-0.18 \\
(-0.81)\end{array}$ & $\begin{array}{c}0.083 \\
(0.074)\end{array}$ & -2.2 & $\begin{array}{c}0.03 \\
(0.016)\end{array}$ & $\begin{array}{c}-0.24 \\
(-0.27)\end{array}$ \\
\hline \multirow[t]{2}{*}{ Interval-Mean } & N1 & $\begin{array}{l}0.035 \\
(0.04)\end{array}$ & $\begin{array}{c}0.005 \\
(0.004)\end{array}$ & 7 & $\begin{array}{c}1.1 \mathrm{E}-9 \\
(6.7 \mathrm{E}-17)\end{array}$ & $\begin{array}{c}0.78 \\
(0.95)\end{array}$ \\
\hline & P2 & $\begin{array}{c}0.002 \\
(-0.002)\end{array}$ & $\begin{array}{c}0.006 \\
(0.005)\end{array}$ & 0.4 & $\begin{array}{c}0.69 \\
(0.69)\end{array}$ & $\begin{array}{c}0.04 \\
(-0.04) \\
\end{array}$ \\
\hline \multirow[t]{2}{*}{ Interval-Previous } & N1 & $\begin{array}{c}0.01 \\
(0.02) \\
\end{array}$ & $\begin{array}{c}0.004 \\
(0.004) \\
\end{array}$ & 2.6 & $\begin{array}{c}0.01 \\
(2.2 \mathrm{E}-7) \\
\end{array}$ & $\begin{array}{c}0.29 \\
(-0.59) \\
\end{array}$ \\
\hline & P2 & $\begin{array}{c}0.02 \\
(0.02)\end{array}$ & $\begin{array}{c}0.004 \\
(0.004)\end{array}$ & 4.4 & $\begin{array}{c}2.5 \mathrm{E}-5 \\
(1.7 \mathrm{E}-8)\end{array}$ & $\begin{array}{c}0.5 \\
(0.64)\end{array}$ \\
\hline \multirow[t]{2}{*}{$\begin{array}{l}\text { Interval-Mean * } \\
\text { Interval-Previous }\end{array}$} & N1 & $\begin{array}{c}-0.0004 \\
(-0.0008)\end{array}$ & $\begin{array}{c}0.0003 \\
(0.0002)\end{array}$ & -1.2 & $\begin{array}{c}0.2 \\
(0.001)\end{array}$ & $\begin{array}{c}-0.14 \\
(-0.36) \\
\end{array}$ \\
\hline & P2 & $\begin{array}{c}-0.0003 \\
(-0.0002)\end{array}$ & $\begin{array}{c}0.0003 \\
(0.0002)\end{array}$ & -1.1 & $\begin{array}{c}0.26 \\
(0.33)\end{array}$ & $\begin{array}{c}-0.12 \\
(-0.11)\end{array}$ \\
\hline
\end{tabular}

\begin{tabular}{|l|c|c|c|}
\hline Comparisons between pairs of predictors & $\mathbf{t}(\mathbf{7 8})$ & $\mathbf{p}$-value & $\mathbf{d}$ \\
\hline N1 vs. P2 Interval-Mean & 3.5 & $\begin{array}{c}7.1 \mathrm{E}-4 \\
(2.6 \mathrm{E}-9)\end{array}$ & $\begin{array}{c}0.39 \\
(0.67)\end{array}$ \\
\hline N1 vs. P2 Interval-Previous & -1.2 & $\begin{array}{c}0.21 \\
(0.6)\end{array}$ & $\begin{array}{c}-0.12 \\
(-0.05)\end{array}$ \\
\hline N1 Interval-Mean vs. -previous & 4.5 & $\begin{array}{c}2.2 \mathrm{E}-5 \\
(4.7 \mathrm{E}-7)\end{array}$ & $\begin{array}{c}0.5 \\
(0.57)\end{array}$ \\
\hline P2 Interval-Mean vs. -previous & -3.3 & $\begin{array}{c}1.3 \mathrm{E}-3 \\
(3.7 \mathrm{E}-6)\end{array}$ & $\begin{array}{c}-0.37 \\
(-0.52)\end{array}$ \\
\hline $\begin{array}{l}\text { N1 Interval-Mean vs. -previous } \\
\text { vs. } \\
\text { P2 Interval-Mean vs. -previous }\end{array}$ & 4.7 & $\begin{array}{c}9.4 \mathrm{E}-6 \\
(1 \mathrm{E}-11)\end{array}$ & $\begin{array}{c}0.53 \\
(0.77)\end{array}$ \\
\hline
\end{tabular}


bioRxiv preprint doi: https://doi.org/10.1101/2020.06.08.141044; this version posted December 9, 2020. The copyright holder for this preprint (which was not certified by peer review) is the author/funder, who has granted bioRxiv a license to display the preprint in perpetuity. It is made available under aCC-BY-NC-ND 4.0 International license.

A

Experiment 1

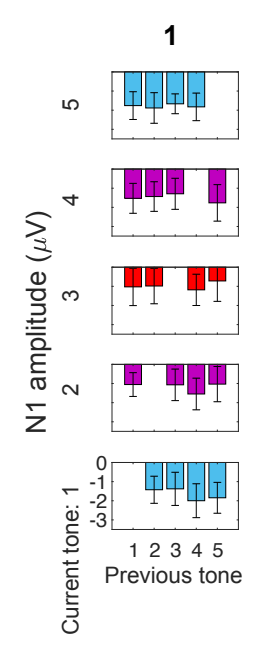

B

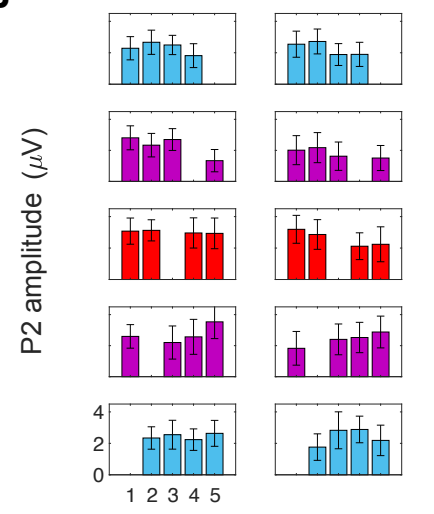

Experiment 2
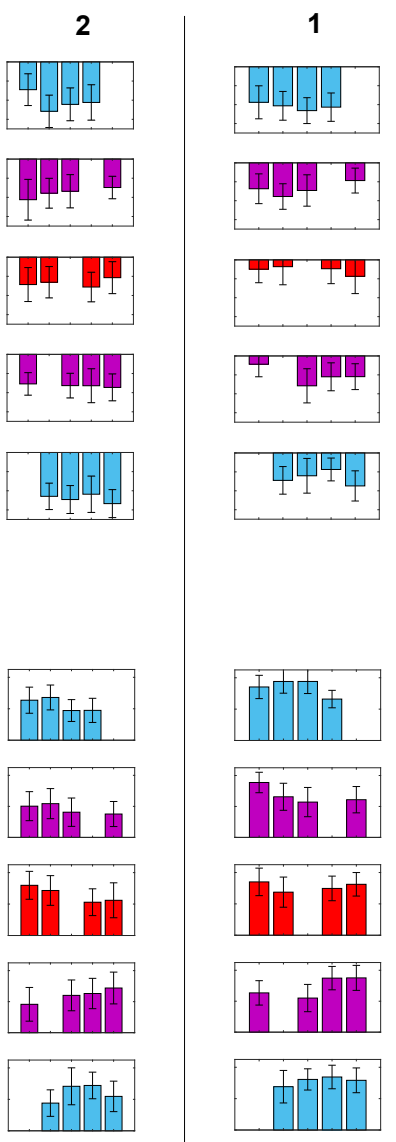

1
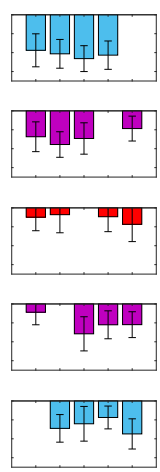

942

943

944

945

946

947

948

949

950

951

952

953

954

955

956

957

958 methods.
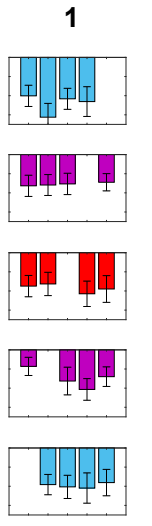

Wide
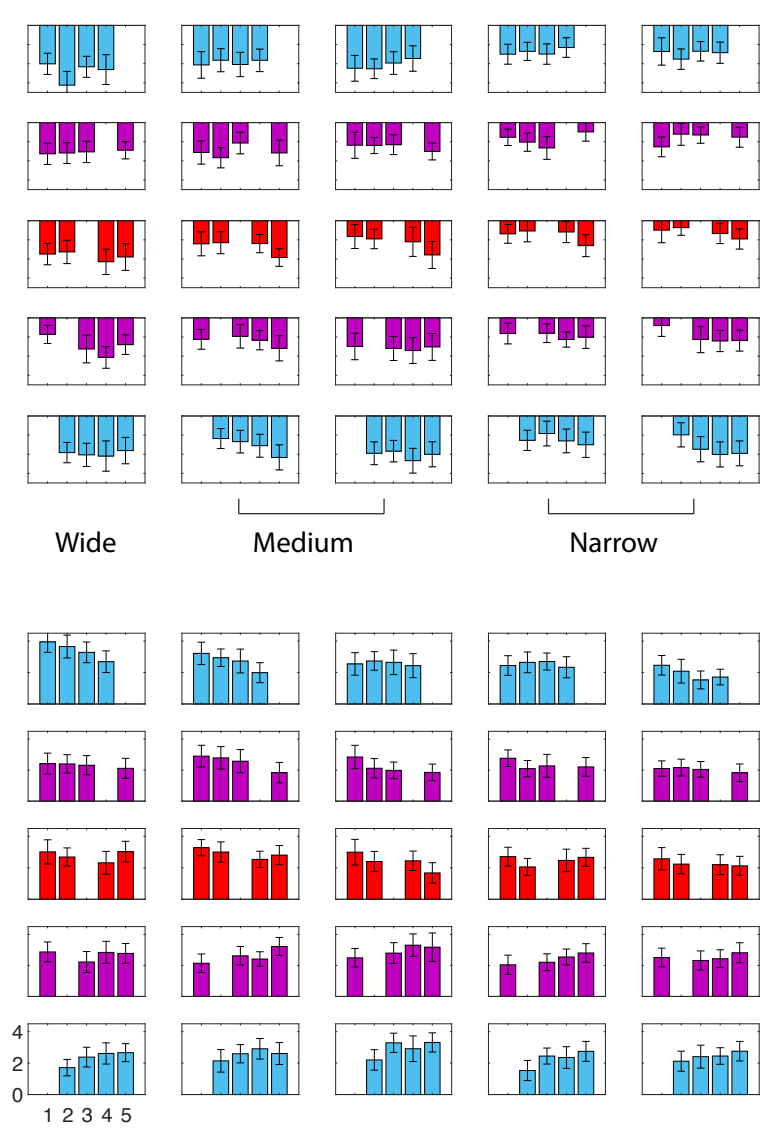

Supplementary Figure S2 - Average N1 and P2 amplitudes, all combinations of current and previous tones. Bar graphs depict average N1 (A) or P2 (B) peak amplitudes averaged across participants for every pair of current and previous tones, in every experiment and block type. Error bars represent 95\% confidence intervals of the peaks across participants. Numbers under experiment titles denote block types. Tones are ranked from 1 to 5 from low to high frequency, within each block type (as illustrated in Figure 1A). Each row of bar graphs shows the N1/P2 amplitudes for a specific tone rank contingent on the previous tone rank. Since there were no immediate repetitions of the same tone, each graph misses the bar for the immediate repetition (e.g. tone 5 following tone 5 of a sequence, tone 4 following tone 4, etc.). For specific tones' frequencies see Figure $1 B$ and 
bioRxiv preprint doi: https://doi.org/10.1101/2020.06.08.141044; this version posted December 9,2020 . The copyright holder for this preprint (which was not certified by peer review) is the author/funder, who has granted bioRxiv a license to display the preprint in perpetuity. It is made available under aCC-BY-NC-ND 4.0 International license.

\section{7}

968

969

970

971

972

973

974

975

976

977

Supplementary Table S2 - Linear mixed effects (LME) results for the long-and short-term context predictors 'Interval-Mean' and 'Interval-Previous', excluding their interaction term. Entries are similar to Table 1. The actual values of Table 1 (from the LME that included the interaction term) are added in parenthesis for comparison. Note that the main difference between these results and those presented in Table 2 is that in this case the contribution of the short-term context variable (Interval-Previous) to N1 (but not P2) was reduced, and as a result the contrast between Interval-Previous contributions to $N 1$ and $P 2$ is larger and significant (highlighted with underline).

\begin{tabular}{|c|c|c|c|c|c|c|}
\hline Predictors & & Estimate & SE & $F(1,8954)$ & p-value & d \\
\hline \multirow[t]{2}{*}{ Intercept } & N1 & $\begin{array}{c}-0.4 \\
(-0.51)\end{array}$ & $\begin{array}{c}0.07 \\
(0.078)\end{array}$ & $32.3(42.2)$ & $\begin{array}{c}1.4 \mathrm{E}-08 \\
(8.8 \mathrm{E}-11)\end{array}$ & $\begin{array}{c}-0.6 \\
(-0.73)\end{array}$ \\
\hline & $\mathbf{P 2}$ & $\begin{array}{c}-0.14 \\
(-0.18)\end{array}$ & $\begin{array}{c}0.06 \\
(0.074)\end{array}$ & $4.9(5.80)$ & $\begin{array}{c}0.028 \\
(0.016)\end{array}$ & $\begin{array}{c}-0.2 \\
(-0.27)\end{array}$ \\
\hline \multirow[t]{2}{*}{ Interval-Mean } & N1 & $\begin{array}{c}0.03 \\
(0.04) \\
\end{array}$ & $\begin{array}{c}0.003 \\
(0.004)\end{array}$ & $83(70.0)$ & $\begin{array}{c}8.2 \mathrm{E}-20 \\
(6.7 \mathrm{E}-17)\end{array}$ & $\begin{array}{c}1 \\
(0.94) \\
\end{array}$ \\
\hline & $\mathbf{P 2}$ & $\begin{array}{c}-0.006 \\
(-0.002)\end{array}$ & $\begin{array}{c}0.004 \\
(0.005)\end{array}$ & $2.3(0.15)$ & $\begin{array}{l}0.13 \\
(0.7)\end{array}$ & $\begin{array}{c}-0.2 \\
(-0.04)\end{array}$ \\
\hline \multirow[t]{2}{*}{ Interval-Previous } & N1 & $\frac{0.009}{(0.01)}$ & $\begin{array}{c}0.002 \\
(0.003)\end{array}$ & $20.4(26.9)$ & $\begin{array}{c}6.4 \mathrm{E}-06 \\
(2.2 \mathrm{E}-07)\end{array}$ & $\begin{array}{c}0.5 \\
(0.58)\end{array}$ \\
\hline & $\mathbf{P 2}$ & $\frac{0.018}{(0.02)}$ & $\begin{array}{c}0.002 \\
(0.003)\end{array}$ & $66(31.8)$ & $\begin{array}{c}5 \mathrm{E}-16 \\
(1.7 \mathrm{E}-08)\end{array}$ & $\begin{array}{c}0.9 \\
(0.63)\end{array}$ \\
\hline
\end{tabular}

\begin{tabular}{|l|l|l|l|}
\hline Comparisons between pairs of predictors & $\mathrm{F}(1,8954)$ & p-value & $\mathrm{d}$ \\
\hline N1 vs. P2 Interval-Mean & $50(35.5)$ & $\begin{array}{l}1.9 \mathrm{E}-12 \\
(2.6 \mathrm{E}-09)\end{array}$ & $\begin{array}{l}0.8 \\
(0.67)\end{array}$ \\
\hline N1 vs. P2 Interval-Previous & $\underline{9(0.23)}$ & $\frac{0.0025}{(0.62)}$ & $\frac{-0.34}{(-0.05)}$ \\
\hline N1 Interval-Mean vs. -previous & $21(25)$ & $\begin{array}{l}4.3 \mathrm{E}-06 \quad(4.6 \mathrm{E}- \\
07)\end{array}$ & $\begin{array}{l}0.52 \\
(0.57)\end{array}$ \\
\hline P2 Interval-Mean vs. -previous & & $\begin{array}{l}1.4 \mathrm{E}-06 \quad(3.7 \mathrm{E}- \\
0\end{array}$ & $\begin{array}{l}-0.54 \\
(-0.52)\end{array}$ \\
\hline $\begin{array}{l}\text { N1 Interval-Mean vs. -previous } \\
\text { vs. } \\
\text { P2 Interval-Mean vs. -previous }\end{array}$ & $23(21)$ & $06)$ & $\begin{array}{l}0.75 \\
(0.77)\end{array}$ \\
\hline
\end{tabular}


bioRxiv preprint doi: https://doi.org/10.1101/2020.06.08.141044; this version posted December 9,2020 . The copyright holder for this preprint (which was not certified by peer review) is the author/funder, who has granted bioRxiv a license to display the preprint in perpetuity. It is made available under aCC-BY-NC-ND 4.0 International license.

Supplementary Table 53 - Linear mixed effects (LME) results including interactions with frequency range and the interaction term Interval-Mean*interval_previous. This Table is almost identical to Table 2, but the LME model included the interaction terms Interval-Mean * Interval-Previous for N1 and P2. Note that the IntervalMean * Interval-Previous terms had no significant contribution (highlighted with underline) and therefore were omitted from the LME model reported in Table 2. However, they affected the statistical comparisons between estimates (lower Table). In parenthesis - values from Table 2 (when relevant) for comparison.

\begin{tabular}{|c|c|c|c|c|c|c|}
\hline \multicolumn{2}{|l|}{ Predictors } & Estimate & SE & $F(1,6186)$ & $p$-value & d \\
\hline \multirow[t]{2}{*}{ Intercept } & N1 & $\begin{array}{l}-0.76 \\
(-0.76)\end{array}$ & $\begin{array}{l}0.11 \\
(0.1)\end{array}$ & $\begin{array}{c}52 \\
(55)\end{array}$ & $\begin{array}{c}6.3 \mathrm{E}-13 \\
(1.2 \mathrm{E}-13) \\
\end{array}$ & $\begin{array}{l}-1.3 \\
(-1.4) \\
\end{array}$ \\
\hline & $\mathbf{P 2}$ & $\begin{array}{l}-0.39 \\
(-0.36)\end{array}$ & $\begin{array}{c}0.12 \\
(0.11)\end{array}$ & $\begin{array}{l}10.7 \\
(9.7)\end{array}$ & $\begin{array}{c}0.001 \\
(0.002)\end{array}$ & $\begin{array}{l}-0.60 \\
(-0.57)\end{array}$ \\
\hline \multirow[t]{2}{*}{ Interval-Mean } & N1 & $\begin{array}{c}0.054 \\
(0.054)\end{array}$ & $\begin{array}{c}0.01 \\
(0.01)\end{array}$ & $\begin{array}{c}17.2 \\
(17.3)\end{array}$ & $\begin{array}{c}3.3 \mathrm{E}-5 \\
(3.2 \mathrm{E}-5)\end{array}$ & $\begin{array}{l}0.76 \\
(0.76)\end{array}$ \\
\hline & $\mathbf{P 2}$ & $\begin{array}{l}-0.029 \\
(-0.028)\end{array}$ & $\begin{array}{c}0.014 \\
(0.014)\end{array}$ & $\begin{array}{c}4.5 \\
(4.2)\end{array}$ & $\begin{array}{c}0.035 \\
(0.041)\end{array}$ & $\begin{array}{c}-0.39 \\
(-0.37)\end{array}$ \\
\hline \multirow[t]{2}{*}{ Interval-Previous } & N1 & $\begin{array}{c}0.027 \\
(0.027)\end{array}$ & $\begin{array}{c}0.009 \\
(0.009)\end{array}$ & $\begin{array}{c}8.7 \\
(8.8)\end{array}$ & $\begin{array}{c}3.2 \mathrm{E}-3 \\
(3.0 \mathrm{E}-3)\end{array}$ & $\begin{array}{c}0.54 \\
(0.54)\end{array}$ \\
\hline & $\mathbf{P 2}$ & $\begin{array}{c}0.042 \\
(0.043)\end{array}$ & $\begin{array}{c}0.009 \\
(0.009)\end{array}$ & $\begin{array}{c}19.7 \\
(21.2)\end{array}$ & $\begin{array}{c}9.0 \mathrm{E}-6 \\
(4.1 \mathrm{E}-6)\end{array}$ & $\begin{array}{c}0.81 \\
(0.84)\end{array}$ \\
\hline \multirow{2}{*}{$\begin{array}{l}\text { Interval-Mean * Interval- } \\
\text { Previous }\end{array}$} & N1 & 0.00002 & 0.0005 & 0.0014 & 0.97 & 0.01 \\
\hline & $\mathbf{P 2}$ & $\underline{0.0005}$ & $\underline{0.0005}$ & $\underline{1.3}$ & $\underline{0.25}$ & $\underline{0.21}$ \\
\hline \multirow[t]{2}{*}{ Range * Intercept } & N1 & $\begin{array}{c}0.019 \\
(0.018)\end{array}$ & $\begin{array}{c}0.004 \\
(0.003)\end{array}$ & $\begin{array}{c}17.5 \\
(36.2)\end{array}$ & $\begin{array}{c}2.9 \mathrm{E}-5 \\
(1.9 \mathrm{E}-9)\end{array}$ & $\begin{array}{l}0.76 \\
(1.1)\end{array}$ \\
\hline & $\mathbf{P 2}$ & $\begin{array}{c}0.008 \\
(0.0041)\end{array}$ & $\begin{array}{c}0.004 \\
(0.003)\end{array}$ & $\begin{array}{l}3.07 \\
(1.8)\end{array}$ & $\begin{array}{c}0.08 \\
(0.18)\end{array}$ & $\begin{array}{c}0.32 \\
(0.24) \\
\end{array}$ \\
\hline \multirow[t]{2}{*}{ Range * Interval-Mean } & N1 & $\begin{array}{c}-0.0011 \\
(-0.0011) \\
\end{array}$ & $\begin{array}{c}0.0004 \\
(0.0004) \\
\end{array}$ & $\begin{array}{l}6.08 \\
(7.2) \\
\end{array}$ & $\begin{array}{c}0.014 \\
(0.007) \\
\end{array}$ & $\begin{array}{c}-0.45 \\
(-0.49) \\
\end{array}$ \\
\hline & $\mathbf{P 2}$ & $\begin{array}{c}0.0004 \\
(0.0006)\end{array}$ & $\begin{array}{c}0.0004 \\
(0.0004)\end{array}$ & $\begin{array}{l}0.97 \\
(2.5)\end{array}$ & $\begin{array}{c}0.32 \\
(0.11)\end{array}$ & $\begin{array}{c}0.18 \\
(0.29) \\
\end{array}$ \\
\hline \multirow[t]{2}{*}{ Range * Interval-Previous } & N1 & $\begin{array}{c}-0.0006 \\
(-0.0006) \\
\end{array}$ & $\begin{array}{l}0.0003 \\
(0.0003) \\
\end{array}$ & $\begin{array}{l}3.2 \\
(4) \\
\end{array}$ & $\begin{array}{c}0.073 \\
(0.047) \\
\end{array}$ & $\begin{array}{c}-0.33 \\
(-0.36) \\
\end{array}$ \\
\hline & $\mathbf{P 2}$ & $\begin{array}{c}-0.0009 \\
(-0.0007)\end{array}$ & $\begin{array}{c}0.0003 \\
(0.0003)\end{array}$ & $\begin{array}{c}7.5 \\
(6.2)\end{array}$ & $\begin{array}{c}6.0 \mathrm{E}-3 \\
(1.2 \mathrm{E}-2) \\
\end{array}$ & $\begin{array}{c}-0.50 \\
(-0.46)\end{array}$ \\
\hline \multicolumn{4}{|c|}{ Comparisons between pairs of predictors } & $F(1,6186)$ & p-value & d \\
\hline \multicolumn{4}{|c|}{ N1 vs. P2 (Range * Intercept) } & $\begin{array}{c}2.95 \\
(10.9)\end{array}$ & $\begin{array}{c}0.086 \\
(0.0009) \\
\end{array}$ & $\begin{array}{l}0.31 \\
(0.6)\end{array}$ \\
\hline \multicolumn{4}{|c|}{ N1 vs. P2 (Range * Interval-Mean) } & $\begin{array}{c}5.96 \\
(9)\end{array}$ & $\begin{array}{c}0.015 \\
(0.002)\end{array}$ & $\begin{array}{c}-0.45 \\
(-0.55)\end{array}$ \\
\hline \multicolumn{4}{|c|}{ N1 vs. P2 (Range * Interval-Previous) } & $\begin{array}{c}0.46 \\
(0.13)\end{array}$ & $\begin{array}{c}0.5 \\
(0.72) \\
\end{array}$ & $\begin{array}{c}0.12 \\
(-0.07)\end{array}$ \\
\hline
\end{tabular}


bioRxiv preprint doi: https://doi.org/10.1101/2020.06.08.141044; this version posted December $9,2020$. The copyright holder for this preprint (which was not certified by peer review) is the author/funder, who has granted bioRxiv a license to display the preprint in perpetuity. It is made available under aCC-BY-NC-ND 4.0 International license.

A
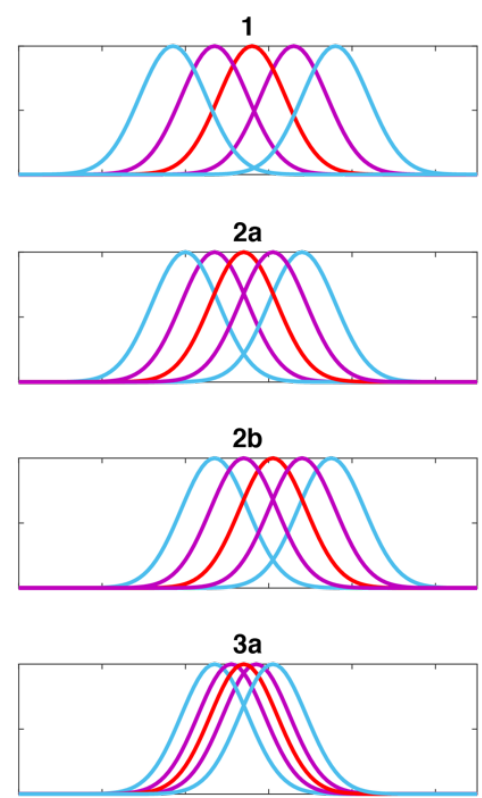

3b

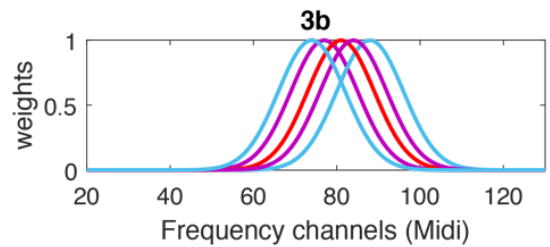

B

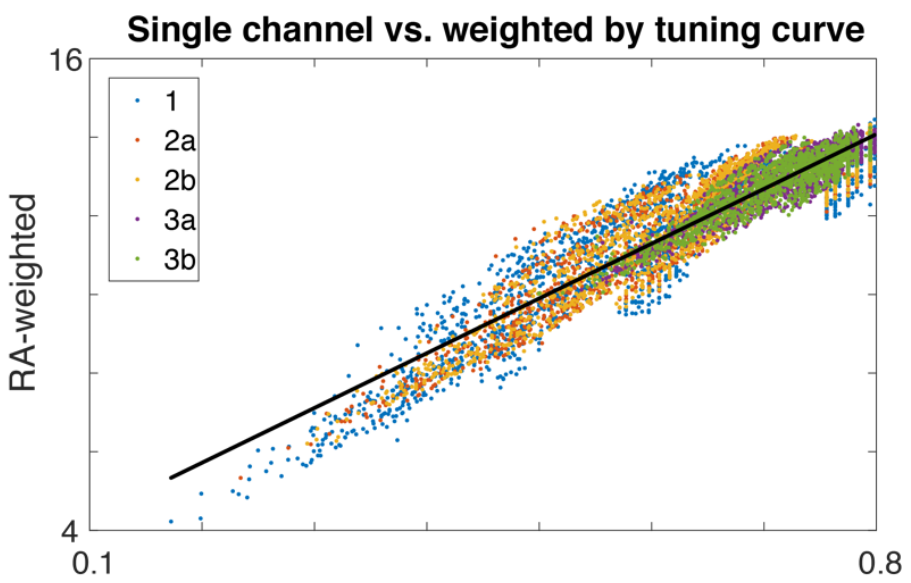

RA

Supplementary Figure S3 - Comparison of RA (the response adaptation model used in this work) to RA weighted by tuning curves. See Methods for a definition of RA. A - Gaussian tuning curves. Each plot corresponds to a specific block type in Experiment 3 (specified in the titles). In each plot, each Gaussian describes the relevant weights for summing up contributions from neural populations with best frequencies displayed along the $x$-axis. The 5 Gaussians correspond to the computed responses to each of the 5 tones, color code similar to Figure 1 . B

- Scatter plot of the weighted RA values (y axis) versus single channel RA values ( $x$ axis) as used in this work following Herrmann et al. (2013, 2014, 2015). 
N1
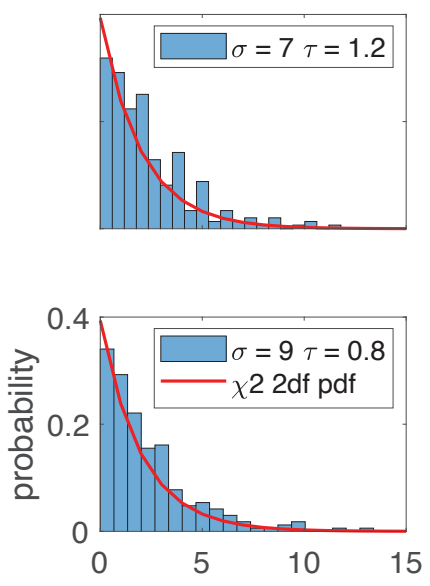

$D$ value

\section{P2}
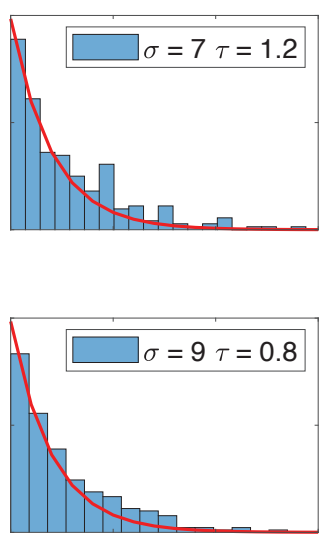

Supplementary Figure S4 - Comparison of the null D distribution to chi-square with 2 degrees of freedom. Blue - histograms of null $D$ distribution computed for 2 representative values of $\vec{\theta}: \sigma=7$ semitones and $\tau=1.2$ seconds (upper) or $\sigma=9$ semitones and $\tau=0.8$ seconds (lower) using $N 1$ (left) or P2 (right) data. Null distribution was simulated by fitting the model to scrambled data, such that the order of data trials did not match that of model predictions, 250 times. The red curve is the theoretical $\chi^{2}$ distribution with 2 degrees-of-freedom.

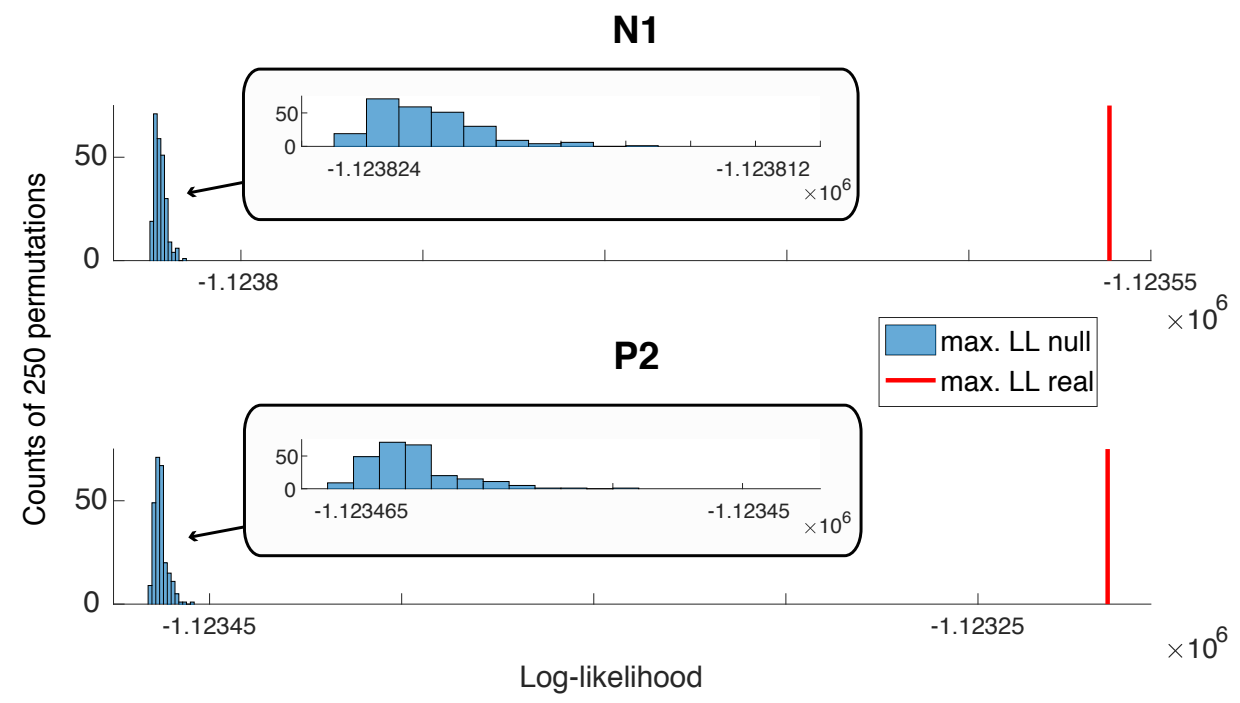

Supplementary Figure S5 - General significance of model fit. Blue bars - Histograms of null distributions of the maximal Log-Likelihood (LL) of LME model fit. Histograms are constructed using 250 repetitions of model fit after permuting the order of data trials, using the data from all experiments. Red lines - the maximal value of $L L$ out of all tested $\vec{\theta}$ values (the LL value at $\vec{\theta}^{\text {max }}$ ), when fitting the model using the real (non-scrambled) data. Top The model fitted to N1 data trials. Bottom - The model fitted to P2 data trials. Insets - Magnifying x-axis values. 

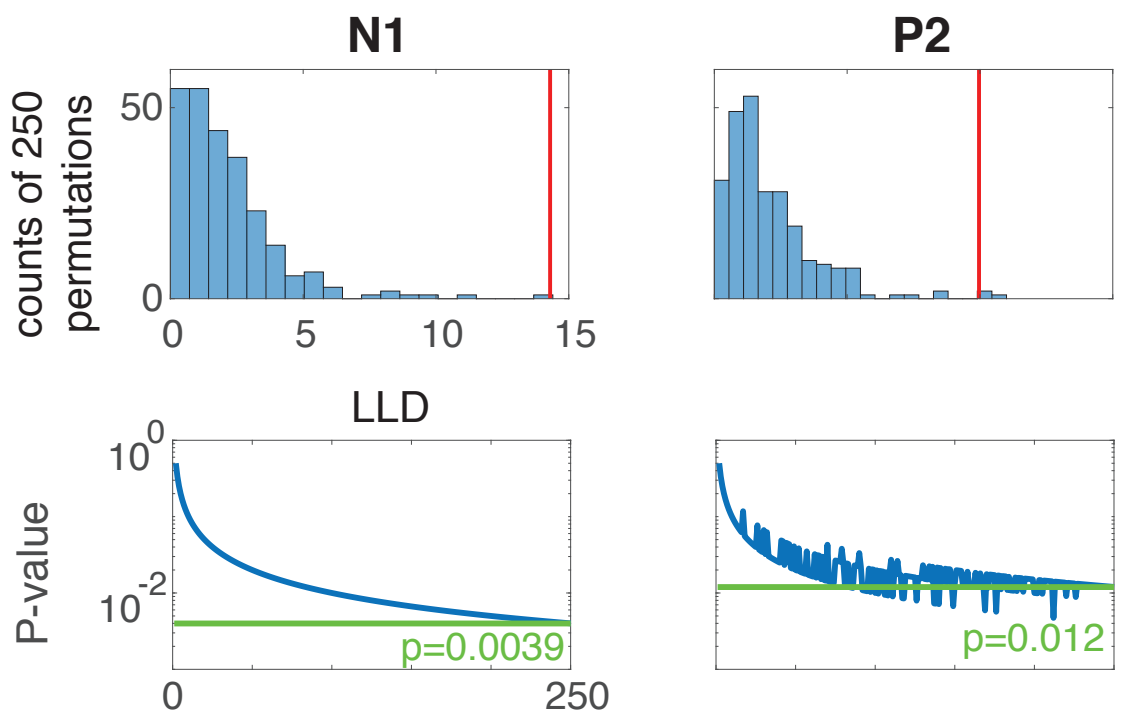

Number of permutations

Supplementary Figure S6 - Differences between log-likelihood at $\tau^{\max }$ and $\tau^{\max }$ of other potential type (LLD) relative to null distribution. Null distribution was calculated by fitting the model and estimating parameters for 250 repetitions of random scrambling of data trials such that their order did not match model predictions. Top blue histograms illustrate the null LLD distribution calculated using the 250 repetitions and red lines indicate the real LLD. Bottom -The green displayed value of $p$ is calculated using all 250 repetitions for the null distribution. The blue line illustrates the $p$-value as a function of the number of repetitions used (scramble groups are selected randomly from the pool of 250 scramble repetitions calculated). The green line denotes the $p$-values calculated when using 250 repetitions. Note that the p-values seem to be an upper bound of the real p-value. Left - N1. 


\section{References}

Ahissar M, Hochstein S (2004) The reverse hierarchy theory of visual perceptual learning. Trends Cogn Sci 8:457-464.

Albrecht AR, Scholl BJ (2010) Perceptually Averaging in a Continuous Visual World. Psychol Sci 21:560-567.

Albrecht AR, Scholl BJ, Chun MM (2012) Perceptual averaging by eye and ear: Computing summary statistics from multimodal stimuli. Attention, Perception, Psychophys 74:810-815.

Arnsten AFT, Paspalas CD, Gamo NJ, Yang Y, Wang M (2010) Dynamic network connectivity: A new form of neuroplasticity. Trends Cogn Sci 14:365-375.

Bar M (2006) Top down facilitation of visual recognition. Proc Natl Acad Sci U S A 103:449454.

Bekinschtein TA, Dehaene S, Rohaut B, Tadel F, Cohen L, Naccache L (2009) Neural signature of the conscious processing of auditory regularities. Proc Natl Acad Sci U S A 106:16721677.

Blake DT, Merzenich MM (2002) Changes of Al receptive fields with sound density. J Neurophysiol 88:3409-3420.

Brenner N, Bialek W, De Ruyter Van Steveninck R (2000) Adaptive rescaling maximizes information transmission. Neuron 26:695-702.

Costa-Faidella J, Grimm S, Slabu L, Díaz-Santaella F, Escera C (2011) Multiple time scales of adaptation in the auditory system as revealed by human evoked potentials. Psychophysiology 48:774-783.

Crowley KE, Colrain IM (2004) A review of the evidence for P2 being an independent component process: Age, sleep and modality. Clin Neurophysiol 115:732-744.

Dahmen JC, Keating P, Nodal FR, Schulz AL, King AJ (2010) Adaptation to Stimulus Statistics in the Perception and Neural Representation of Auditory Space. Neuron 66:937-948.

Davis PA (1939) Effects of acoustic stimuli on the waking human brain. J Neurophysiol 2:494-499.

Dean I, Robinson BL, Harper NS, McAlpine D (2008) Rapid neural adaptation to sound level statistics. J Neurosci 28:6430-6438.

Dunn FA, Rieke F (2006) The impact of photoreceptor noise on retinal gain controls. Curr Opin Neurobiol 16:363-370.

Dürschmid S, Edwards E, Reichert C, Dewar C, Hinrichs H, Heinze H-J, Kirsch HE, Dalal SS, Deouell LY, Knight RT (2016) Hierarchy of prediction errors for auditory events in human temporal and frontal cortex. Proc Natl Acad Sci 113:6755-6760.

Fairhall AL, Lewen GD, Bialek W, de Ruyter van Steveninck RR (2001) Efficiency and ambiguity in an adaptive neural code. Nature 412:787-792.

Garrido MI, Sahani M, Dolan RJ (2013) Outlier Responses Reflect Sensitivity to Statistical 
Structure in the Human Brain. PLoS Comput Biol 9.

Gourévitch B, Noreña A, Shaw G, Eggermont JJ (2009) Spectrotemporal receptive fields in anesthetized cat primary auditory cortex are context dependent. Cereb Cortex 19:1448-1461.

Haberman J, Harp T, Whitney D (2009) Averaging facial expression over time. J Vis 9:1-1.

Hari R, Kaila K, Katila T, Tuomisto T, Varpula T (1982) Interstimulus interval dependence of the auditory vertex response and its magnetic counterpart: Implications for their neural generation. Electroencephalogr Clin Neurophysiol 54:561-569.

Herrmann B, Augereau T, Johnsrude IS (2020) Neural Responses and Perceptual Sensitivity to Sound Depend on Sound-Level Statistics. Sci Rep 10:1-12.

Herrmann B, Henry MJ, Fromboluti EK, McAuley JD, Obleser J (2015) Statistical context shapes stimulus-specific adaptation in human auditory cortex. J Neurophysiol 113:2582-2591.

Herrmann B, Henry MJ, Johnsrude IS, Obleser J (2016) Altered temporal dynamics of neural adaptation in the aging human auditory cortex. Neurobiol Aging.

Herrmann B, Henry MJ, Obleser J (2013) Frequency-specific adaptation in human auditory cortex depends on the spectral variance in the acoustic stimulation. J Neurophysiol 109:2086-2096.

Herrmann B, Maess B, Johnsrude IS (2018) Aging affects adaptation to sound-level statistics in human auditory cortex. J Neurosci 38:1989-1999.

Herrmann B, Schlichting N, Obleser J (2014) Dynamic Range Adaptation to Spectral Stimulus Statistics in Human Auditory Cortex. J Neurosci 34:327-331.

Hollingworth HL (1910) The Central Tendency of Judgment. J Philos Psychol Sci Methods 7:461.

Jaffe-Dax S, Frenkel O, Ahissar M (2017) Dyslexics' faster decay of implicit memory for sounds and words is manifested in their shorter neural adaptation. Elife 6.

Jung T-P, Makeig S, Humphries C, Lee T-W, McKeown MJ, Iragui V, Sejnowski TJ (2000) Removing electroencephalographic artifacts by blind source separation. Psychophysiology 37:163-178.

Khouri L, Nelken I (2015) Detecting the unexpected. Curr Opin Neurobiol 35:142-147.

Knight RT, Hillyard SA, Woods DL, Neville HJ (1980) The effects of frontal and temporalparietal lesions on the auditory evoked potential in man. Electroencephalogr Clin Neurophysiol 50:112-124.

Koelsch S (2009) Music-syntactic processing and auditory memory: Similarities and differences between ERAN and MMN. Psychophysiology 46:179-190.

Kvale MN, Schreiner CE (2004) Short-Term Adaptation of Auditory Receptive Fields to Dynamic Stimuli. J Neurophysiol 91:604-612.

Lanting CP, Briley PM, Sumner CJ, Krumbholz K (2013) Mechanisms of adaptation in human 
auditory cortex. J Neurophysiol 110:973-983.

Lee CM, Osman AF, Volgushev M, Escabí MA, Read HL (2016) Neural spike-timing patterns vary with sound shape and periodicity in three auditory cortical fields. J Neurophysiol 115:1886-1904.

Lieder I, Adam V, Frenkel O, Jaffe-Dax S, Sahani M, Ahissar M (2019) Perceptual bias reveals slow-updating in autism and fast-forgetting in dyslexia. Nat Neurosci 22:256-264.

Maheu M, Dehaene S, Meyniel F (2019) Brain signatures of a multiscale process of sequence learning in humans. Elife 8.

Maravall M, Petersen RS, Fairhall AL, Arabzadeh E, Diamond ME (2007) Shifts in coding properties and maintenance of information transmission during adaptation in barrel cortex. PLoS Biol 5:0323-0334.

McDermott JH, Schemitsch M, Simoncelli EP (2013) Summary statistics in auditory perception. Nat Neurosci 16:493-U169.

McDermott JH, Simoncelli EP (2011) Sound texture perception via statistics of the auditory periphery: Evidence from sound synthesis. Neuron 71:926-940.

Nagel KI, Doupe AJ (2006) Temporal Processing and Adaptation in the Songbird Auditory Forebrain. Neuron 51:845-859.

Nahum M, Nelken I, Ahissar M (2008) Low-Level Information and High-Level Perception: The Case of Speech in Noise Poeppel D, ed. PLoS Biol 6:e126.

Okamoto H, Kakigi R (2014) History of silence affects auditory evoked fields regardless of intervening sounds: A magnetoencephalographic study. Eur J Neurosci 40:3380-3386.

Osman AF, Lee CM, Escabí MA, Read HL (2018) A hierarchy of time scales for discriminating and classifying the temporal shape of sound in three auditory cortical fields. J Neurosci 38:6967-6982.

Piazza EA, Sweeny TD, Wessel D, Silver MA, Whitney D (2013) Humans Use Summary Statistics to Perceive Auditory Sequences. Psychol Sci 24:1389-1397.

Picton TW (2011) Human Auditory Evoked Potentials. Plural Publishing.

Poulin-Charronnat B, Bigand E, Koelsch S (2006) Processing of musical syntax tonic versus subdominant: an event-related potential study. J Cogn Neurosci 18:1545-1554.

Rabinowitz NC, Willmore BDB, Schnupp JWH, King AJ (2011) Contrast Gain Control in Auditory Cortex. Neuron 70:1178-1191.

Raviv O, Ahissar M, Loewenstein Y (2012) How Recent History Affects Perception: The Normative Approach and Its Heuristic Approximation Sekuler R, ed. PLoS Comput Biol 8:e1002731.

Regev TI, Nelken I, Deouell LY (2019) Evidence for Linear but Not Helical Automatic Representation of Pitch in the Human Auditory System. J Cogn Neurosci 31:669-685.

Sams M, Hari R, Rif J, Knuutila J (1993) The Human Auditory Sensory Memory Trace Persists about 10 sec: Neuromagnetic Evidence. J Cogn Neurosci 5:363-370. 
Snyder a C, Shpaner M, Molholm S, Foxe JJ (2012) Visual object processing as a function of stimulus energy, retinal eccentricity and Gestalt configuration: a high-density electrical mapping study. Neuroscience 221:1-11.

Sussman E (2007) A New View on the MMN and Attention Debate: The Role of Context in Processing Auditory Events. J Psychophysiol 21:164-175.

1143 Taaseh N, Yaron A, Nelken I (2011) Stimulus-Specific Adaptation and Deviance Detection in the Rat Auditory Cortex. PLoS One 6:e23369.

Tremblay K, Kraus N, McGee T, Ponton C, Otis B (2001) Central auditory plasticity: changes in the N1-P2 complex after speech-sound training. Ear Hear 22:79-90.

Ulanovsky N, Las L, Farkas D, Nelken I (2004) Multiple Time Scales of Adaptation in Auditory Cortex Neurons. J Neurosci 24:10440-10453.

Wilkinson GN, Rogers CE (1973) Symbolic Description of Factorial Models for Analysis of Variance. Appl Stat 22:392.

Zacharias N, König R, Heil P (2012) Stimulation-history effects on the M100 revealed by its differential dependence on the stimulus onset interval. Psychophysiology 49:909-919. 